\title{
HYDROGEN STREAMER CHAMBER UP TO ATMOSPHERIC PRESSURE
}

\author{
F. ROHRBACH, M. CATHENOZ, J. JENNY
}

CERN, Geneva, Switzerland

J. COLAS, D. FOURNIER, J. M. NOPPE and J. J. VEILLET

Laboratoire de I'Accélérateur Linéaire, Orsay, France

Received 15 October 1976

\begin{abstract}
A large hydrogen streamer chamber has been built at CERN $\left(20 \times 35 \times 55 \mathrm{~cm}^{3}\right)$. Good tracks of cosmic rays have been obtained up to atmospheric pressure using an image intensifier. The streamers are small $(<\sim 500 \mu \mathrm{m})$ in diameter, short in length (a few $\mathrm{mm}$ ) and well contrasted. The electric field to obtain tracks at atmospheric pressure is $(66 \pm 3) \mathrm{kV} / \mathrm{cm}$ with a $3 \mathrm{~ns}$ fwhm pulse. An addition of $\sim \frac{1}{2} \% \mathrm{CH}_{4}$ in $\mathrm{H}_{2}$ is necessary in order to avoid parasitic streamers from photoclectrons. The memory of the gas mixed with $\mathrm{ppm}$ of $\mathrm{SF}_{6}$ and the effect of the amplitude jitter on the quality of the tracks have been measured and studied.
\end{abstract}

\section{Introduction}

The hydrogen streamer chamber (HSC) has several attractive features as a detector for high energy physics. Like all streamer chambers it is a triggerable device with a nearly $4 \pi$ acceptance, able to handle up to $\sim 10^{6}$ beam particles per second. In addition the $\mathrm{H}_{2}$ or $\mathrm{D}_{2}$ gas in the chamber acts simultaneously as a target and as a detector for charged particles. HSCs offer the opportunity of seeing the vertex of an interaction on proton or deuteron and of photographing all the charged particle trajectories coming from the interaction (even the short ones) as in a hydrogen bubble chamber (HBC). But due to the low gas density used short tracks in gas mean very low momentum particles: a $20 \mathrm{MeV} / \mathrm{c}$ proton gives $\sim 1 \mathrm{~cm}$ track in the chamber. Such low momenta particles are not detected in a HBC. Due to the low mass of the gas target $(\sim 750$ times smaller than in liquid hydrogen), the multiple scattering is negligible and no secondary interactions limit the length of the tracks; this means that it is possible to make precise measurement of momenta when the chamber is placed in a magnetic field (a track of $1 \mathrm{~m}$ in $30 \mathrm{kG}$ is measured with a $\Delta p / p$ below $1 \%$ from the lowest momentum up to $\sim 10 \mathrm{GeV} / \mathrm{c}$ ). Using the relativistic increase in ionisation particle identification is also possible. These characteristics make HSCs well suited to study problems like diffraction dissociation, Coulomb interference with strong interaction and very low energy interactions. Using a simple trigger on an identified incoming particle making an interaction in the chamber, the HSC can also be used as a bubble chamter in very high energy interactions when a separated beam can no longer be achieved.
Results obtained in 1971 at CERN ${ }^{1}$ ) were sufficiently encouraging to believe that it could be possible to obtain good quality tracks in $\mathrm{H}_{2}$ up to atmospheric pressure. Late in 1973 the technical development work on HSC was resumed in order to prove that belief and to study the feasibility of a physics experiment with such a device.

On the basis of former results ${ }^{1}$ ) and in view of foreseen experiments* (a $2 \times 20 \mathrm{~cm}$ gap streamer chamber was envisaged) a $2 \mathrm{MV}$ Marx generator was designed and built. Preliminary tests were performed in the small $9 \mathrm{~cm}$ single gap streamer chamber used in 1971. Then, a half-scale streamer chamber $(2 \times$ $\times 10 \mathrm{~cm}$ gaps) was built and tested with $\mathrm{H}_{2}$ up to the atmospheric pressure. The results of these tests are presented below. We also point out the main difficulties encountered and the problems which still remain and which must be overcome in order to obtain an operational full scale chamber.

\section{Marx generator}

The $2 \mathrm{MV}$ Marx generator is a 10 stage, $10000 \mathrm{pF} /$ stage, double polarity $\left(U_{0}= \pm 100 \mathrm{kV}\right.$ maximum $)$ voltage multiplication device (see figs. 1 and 2). More than usual care has been taken to control the field

\footnotetext{
* Using $\mathrm{H}_{2}$ and $\mathrm{D}_{2}$ as both a detector and a gas target the study of electromagnetic and strong interactions in the very low momentum transfer region was intended $[0.001<t<0.1$ $\left(\mathrm{GeV} / \mathrm{c}^{2}\right)^{2}$. More precisely the measurement of the radiative decay width $\Gamma \rho \rightarrow \pi \gamma$ by means of the Primakoff effect was envisaged. In such an electromagnetic interaction the recoil proton has a momentum around $\sim 30 \mathrm{MeV} / \mathrm{c}$. This experiment also gives a measurement of the phase of the strong interaction amplitude.
} 
gradient everywhere in the oil tank. The maximum $\mathrm{dc}$ field in the oil is $80 \mathrm{kV} / \mathrm{cm}$ at full designed charging voltage. This electric stress is low for the Shell Diala C oil used which was filtered and treated (water free). The capacitors were specially designed and built for this purpose in collaboration with industry ${ }^{2}$ ). The Marx tube is made of plexiglass. The spark gaps are made from stainless steel hemispheres $(5 \mathrm{~cm}$ diameter) and the 10 gaps are fixed to $10 \mathrm{~mm}$. The maximum value of the field along the inside of the Marx tube is below $20 \mathrm{kV} / \mathrm{cm}$ for the maximum charging voltage. The trigger of the Marx was specially designed following development work previously carried out at the University of Washington by one of $\mathrm{us}^{3}$ ). It consists of a trigatron ring set at half the breakdown voltage of the first Marx stage and put in the medium plane of the first spark gap. The trigatron ring is fired using a $\sim 20 \mathrm{kV}$ pulse produced by a cascade chain built in $\mathrm{Orsay}^{4}$ ).

Fig. 3 shows the dependence of the breakdown voltage with the pressure in the tube and the working conditions. Due to the difficulty encountered in building a large pressurized plexiglass tube the charging voltage has been limited to $80 \mathrm{kV}( \pm 40 \mathrm{kV})$ in pure dry $\mathrm{N}_{2}$ and extended to $90 \mathrm{kV}( \pm 45 \mathrm{kV})$ using a $\mathrm{N}_{2}+10-15 \%$ $\mathrm{SF}_{6}$ mixture.

From the short circuit measurement of the Marx current the value of the equivalent $R_{0}, L_{0}, C_{0}$ Marx circuit parameters have been estimated (see fig. 4 and ref. 2)

$$
C_{0}=490 \mathrm{pF}, \quad L_{0}=(13.4 \pm 0.4) \mu \mathrm{H}, \quad R_{0}=(21 \pm 3) \Omega .
$$

The measured output stray capacitance $C_{\mathrm{s}}$ of the connection to the Blumlein is $150 \mathrm{pF}$. Due to the high impedance of the Marx generator $\left[\sim \sqrt{ }\left(L_{0} / C_{0}\right)=165 \Omega\right]$ a self-inductance must be installed between the Marx output and the Blumlein line load to match the load to the Marx impedance. This self-inductance $L_{\mathrm{s}}$ is made of a few turns of $\mathrm{Cu}$ pipe put in the oil tank and has a value of $\sim 26 \mu \mathrm{H}$. With these elements the ratio between the total dc charging voltage $2 n U_{0}=20 U_{0}$ and the maximum voltage $U_{\mathrm{B} \max }$ on the $3 \mathrm{~ns}$ Blumlein $\left(2 Z_{0}=40 \Omega\right)$ used for the small $9 \mathrm{~cm}$ single gap $40 \Omega$ chamber is $0.9 \pm 0.05$ and $\sim 0.8$ for the $3 \mathrm{~ns} / 25 \Omega$ Blumlein used in the case of the $2 \times 10 \mathrm{~cm}^{2}$ double gap $25 \Omega$ chamber.

The rise time of the Marx voltage output on the Blumlein is $150 \mathrm{~ns}$. The various time delays which

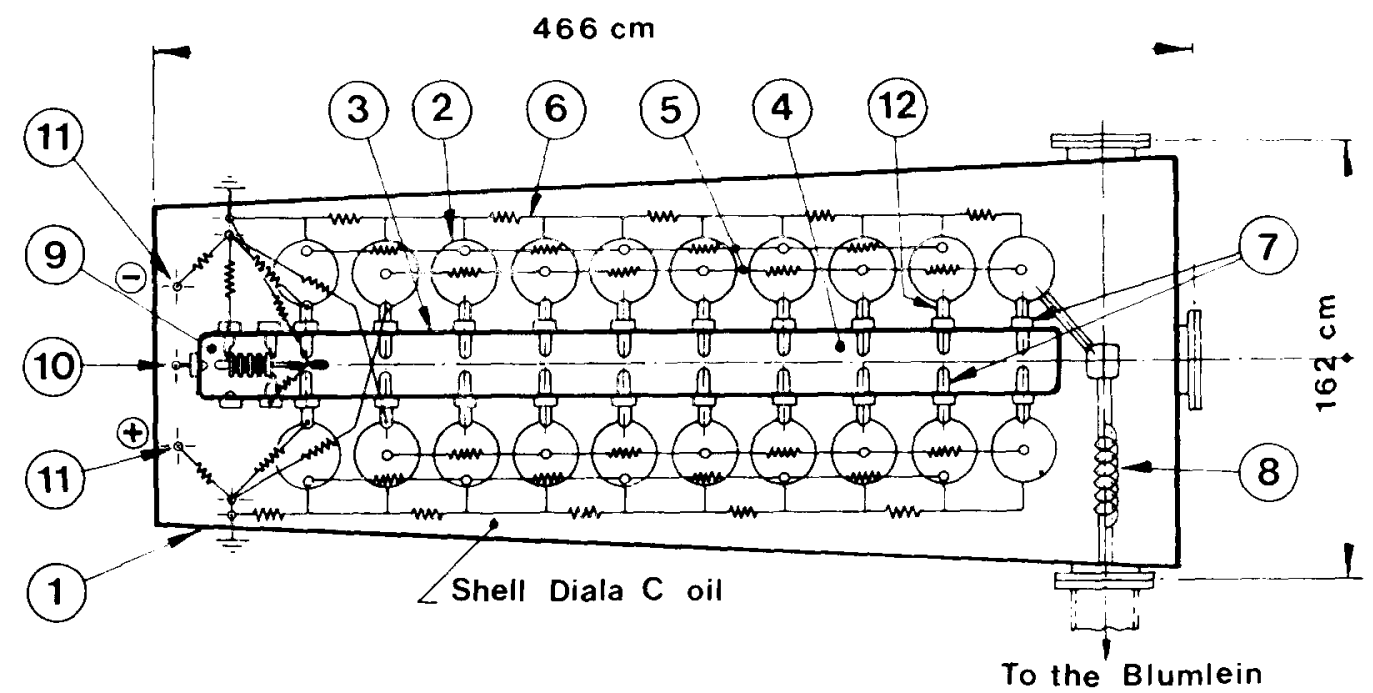

(1) Oil tank

(2) Capacitors Cogeco, $100 \mathrm{kV}, 10 \mathrm{nf}$

(3) Plexiglas Marx tube, $20 \mathrm{~cm}$ diameter

(4) $\mathrm{N}_{2}$ pressurized gas -1 to +2 atm.

(5) Charging $4 \mathrm{k} \Omega$ Carbon resistors

(6) Ground discharge Carbon resistors
(7) Stainless steel spark gaps, $10 \mathrm{~mm}$ gap

(8) Output self inductance $L_{s}=26 \mu \mathrm{H}$

(9) Trigger system

(10 $\odot 20 \mathrm{kV}$ Triggering pulse input

(11) $\oplus$ and $\odot$ D.C. charging voltage

(12) Spark gap to condensor connection

Fig. 1. $2 \mathrm{MV}$ Marx power supply - schematic diagram. 
have been measured for best setting conditions are (see fig. 4):

- Photomultiplier + cable + coincidence logic:

$$
t_{1}=\sim 110 \mathrm{~ns},
$$

- Cable + cascade chain at $20 \mathrm{kV}$ :

$$
t_{2}-t_{1}=\sim 260 \mathrm{~ns} \text {, }
$$

- Trigatron + spark gaps switching:

$$
t_{3}-t_{2}=\sim 220 \mathrm{~ns} \text {, }
$$

- Half period for rising voltage:

$$
t_{4}-t_{3}=\sim 300 \mathrm{~ns} \text {, }
$$

- Switching + transmission to the chamber:

$$
t_{5}-t_{4}=\sim 30 \mathrm{~ns} \text {, }
$$

- Total delay measured:

$$
t_{5} \sim 950 \mathrm{~ns} \text {. }
$$

\section{The Blumlein line (see fig. 5)}

As already shown by previous tests with direct photography ${ }^{1}$ ) the high voltage pulse on the chamber must be very short to produce good streamers in $\mathrm{H}_{2}\left(\tau_{1 / 2} \lesssim 3-4 \mathrm{~ns}\right)^{*}$. A Blumlein line has been built for two values of the pulse length $\left(\tau_{0}=3.1 \text { and } 5.5 \mathrm{~ns}\right)^{*}$ in order to check again the effect of the pulse length on the streamer size and brightness in $\mathrm{H}_{2}$ using an image intensifier camera (IIC) instead of direct photography. Following development work previously carried out at $C E R N^{1}$ ) and at the University of

\footnotetext{
* $\tau_{\frac{1}{2}}$ means the duration of the pulse taken at half its peak value, the so-called fwhm (full width at half maximum), $\tau_{0}$ means the theoretical full width for zero rise time.
}

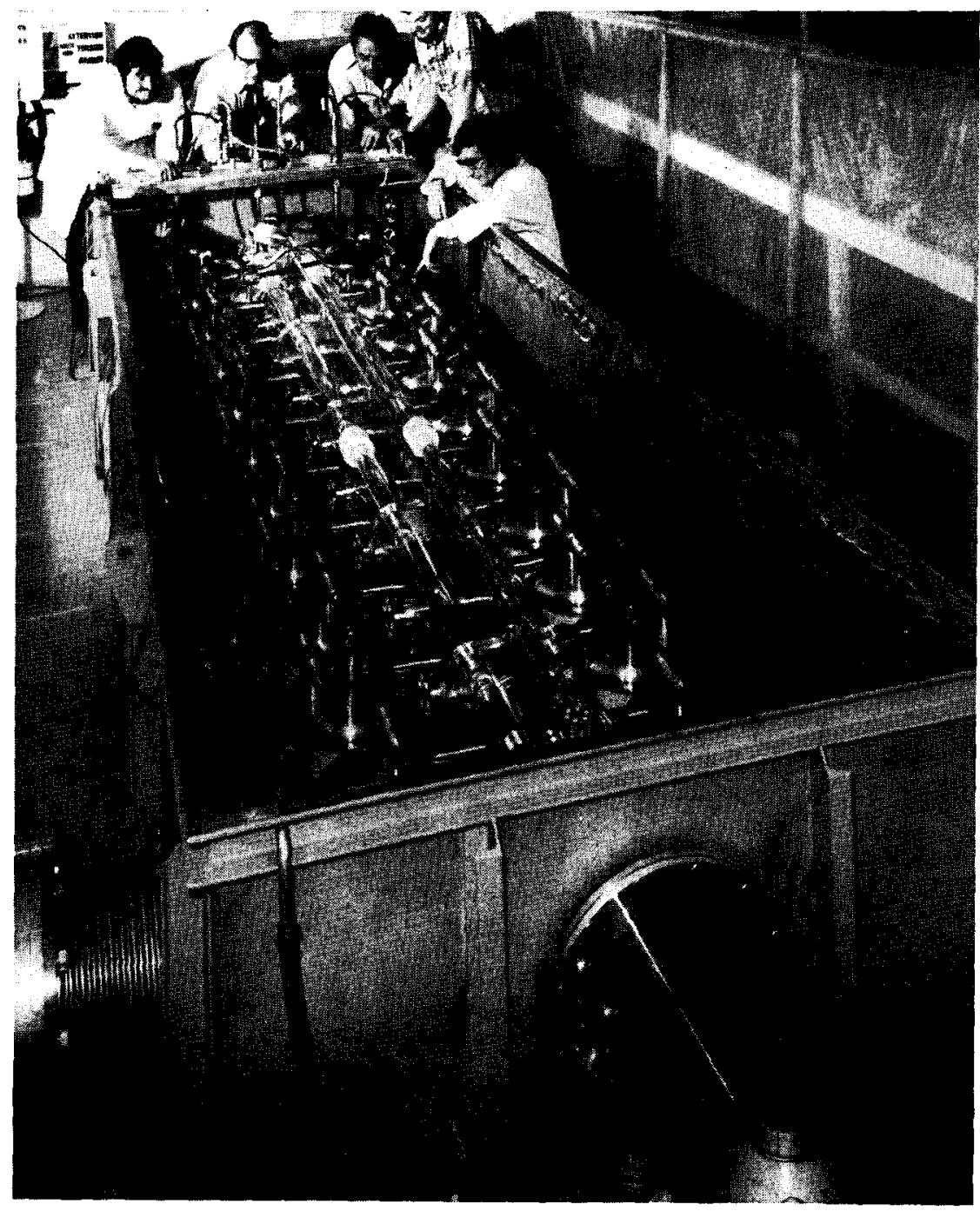

Fig. 2. Inside view of the $2 \mathrm{MV}$ Marx generator. 
Washington $\left.{ }^{5}\right)$ on the generation of very fast $(\sim 1 \mathrm{~ns}$ rise time) high voltage pulses, the Blumlein has been designed as a conical coaxial line with a pressurized spark gap put at the tip of the cone. $\ln$ order to match the $40 \Omega$ impedance of the small single gap chamber and the $25 \Omega$ of the large double gap chamber, two similar conical Blumlein lines have been built. The impedance of each one has been matched to the corresponding chamber by changing the ratio of the various diameters and opening angles keeping constant the external diameter. The conical part of the Blumlein line is elongated by a cylindrical part insulated with oil. By changing the length of the cylindrical part the length of the pulse can be made either 3.1 or $5.5 \mathrm{~ns}$. This line is compensated for the geometrical difference in length by taking advantage of the $\varepsilon$ ratio between gas and oil at the tip of the cone $\left(\varepsilon_{\mathrm{oil}}=2.13 \pm 0.10\right.$,

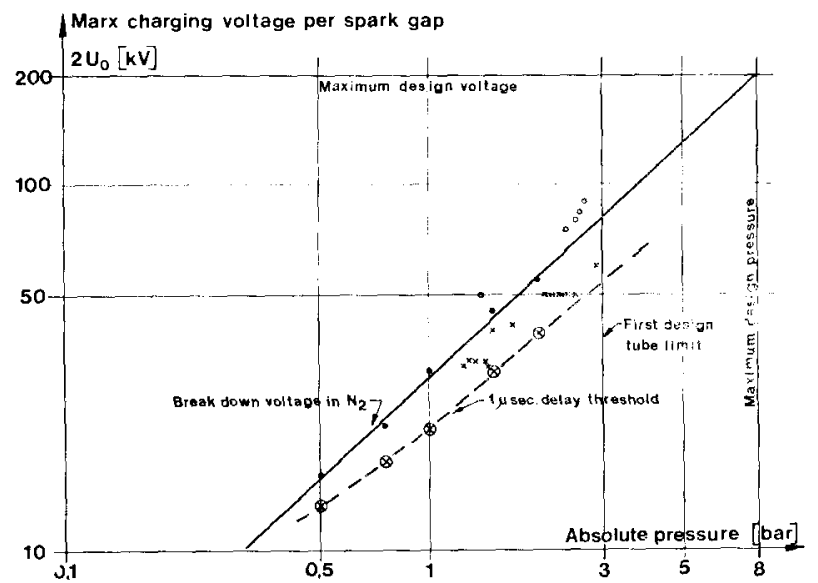

Fig. 3. Dependence of breakdown voltage of the Marx tube on $\mathrm{N}_{2}$ pressure (O) and working conditions in $\mathrm{N}_{2}(\times)$ and with an addition of $10-15 \% \mathrm{SF}_{6}(\mathrm{O})$. The $1 \mu \mathrm{s}$ delay triggering threshold in $\mathrm{N}_{2}$ is also shown $(8)$.

TABLE !

Electric strength of dielectric material in the ns pulse duration range.

Purified Shell Diala $C$ oil

$\mathrm{N}_{2}$ at atmospheric pressure

$\mathrm{SF}_{6}$ at atmospheric pressure

Freon 114 at atmospheric pressure

Araldite

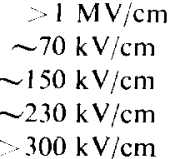

measured). The radius at the tip of the cone has been made small enough so the rise time is limited by the spark characteristics and not by the circuit ${ }^{1}$ ). The gap of the radial spark gap is adjustable from contact to $10 \mathrm{~mm}$. Above $6-7 \mathrm{~mm}$, due to the cylindrical geometry, the jitter of the breakdown voltage increases and the pulse shape changes because the spark does not occur always in the gap but also in the conical region. The spark gap is pressurized with $\mathrm{SF}_{6}$ gas up to a maximum of 15 bar. There is a constant flow $(\sim 11 / \mathrm{min})$ of fresh $\mathrm{SF}_{6}$ in order to remove lower fluorides of sulphur which have lower breakdown voltage than $\mathrm{SF}_{6}$ *.

The size of the cylindrical part of the Blumlein line is determined by the breakdown field of the insulating material used for the bushings and the maximum designed working voltage. In order to use the full electrical strength of the insulators, particular precautions must be taken when designing the bushings in order to avoid reflections giving rise to large overvoltages $^{6}$ ) at the discontinuities between the different materials (gas, araldite, oil); a good solution to this problem is to set the various insulator edges at Brewster

* After a run of about 10000 pulses a grey powder was found in the spark gap. This powder mainly made of fluorides is toxic but it does not change the breakdown voltage of the spark gap.

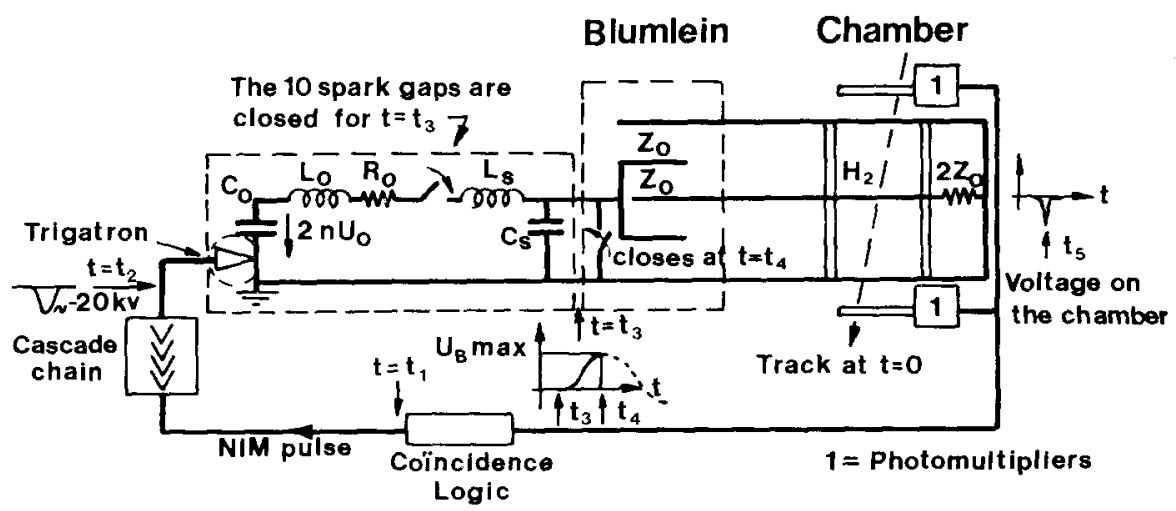

Fig. 4. Schematic of the system showing the equivalent Marx circuit parameters, the main components and defining the delays. 


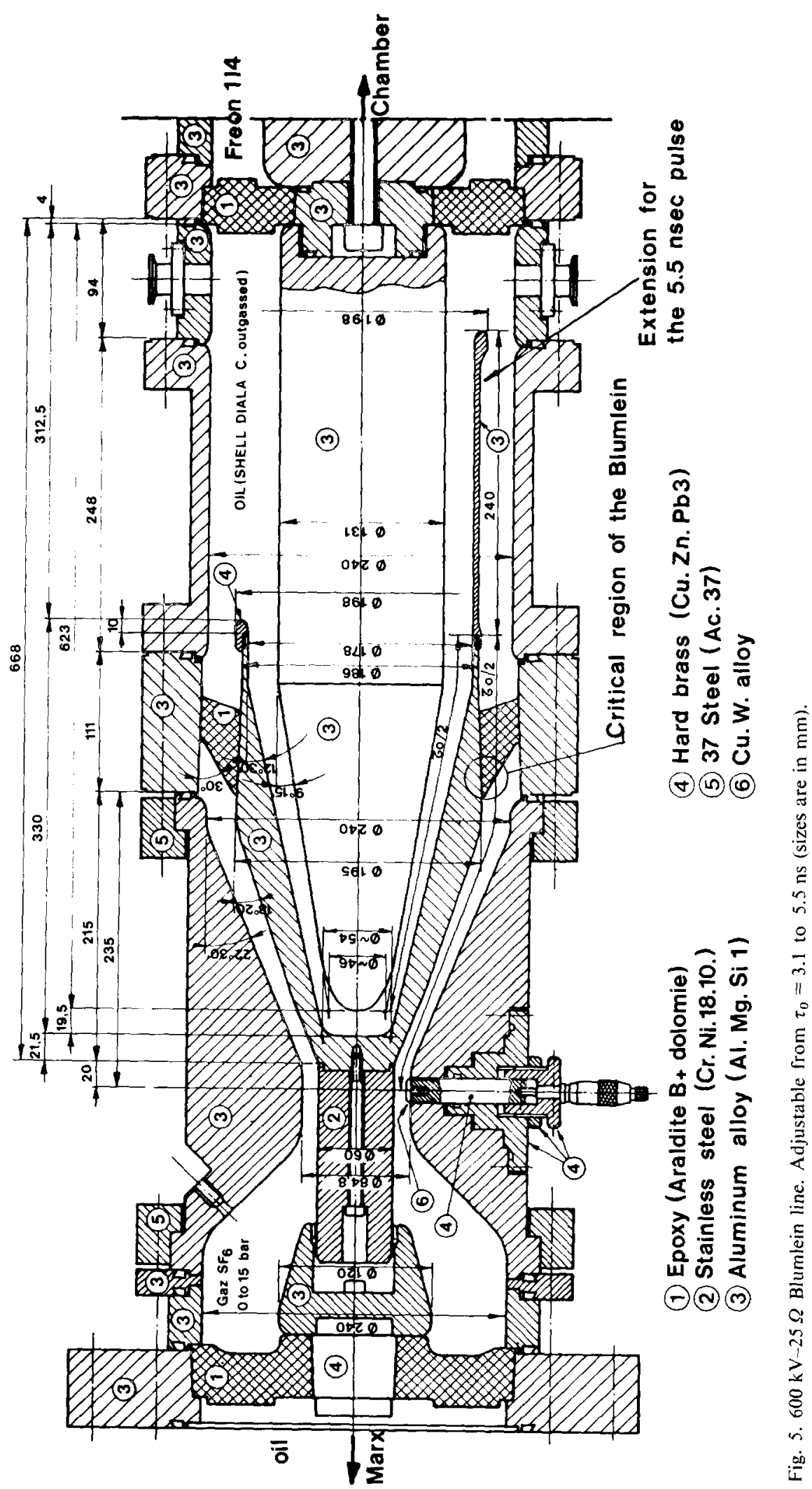


angles. It is also important to avoid all kinds of low $\varepsilon$ dielectric gaps (perfect gluing) across the electric field lines and to shape every metal piece and high dielectric strength material with large radius of curvature in order to control the field everywhere at a value well below the electric strength of the material (see table 1).

These values have been found from tests performed using the $3 \mathrm{~ns}$ half width Blumlein pulse. The results agree with those given by Felsenthal and Proud ${ }^{7}$ ).

The amplitude of the peak voltage of the pulse measured on the chamber is dependent upon the gas pressure and the spark gap width (see fig. 6) and does not depend on first approximation on the maximum amplitude of the ringing charging voltage from the Marx, however, the jitter does. A good setting condition in order to minimise the output amplitude jitter without cutting too much the pulse amplitude is to adjust the gas pressure and/or gap width for obtaining an auto-firing of the Blumlein spark gap at about $10 \%$ below the maximum amplitude of the charging voltage. The best value of the jitter obtained was $4 \%$ (jitter is defined as $2.35 \times$ standard deviation $\sigma$ from the mean; this is almost equal to the fwhm for most of the pulse height distributions which are close to

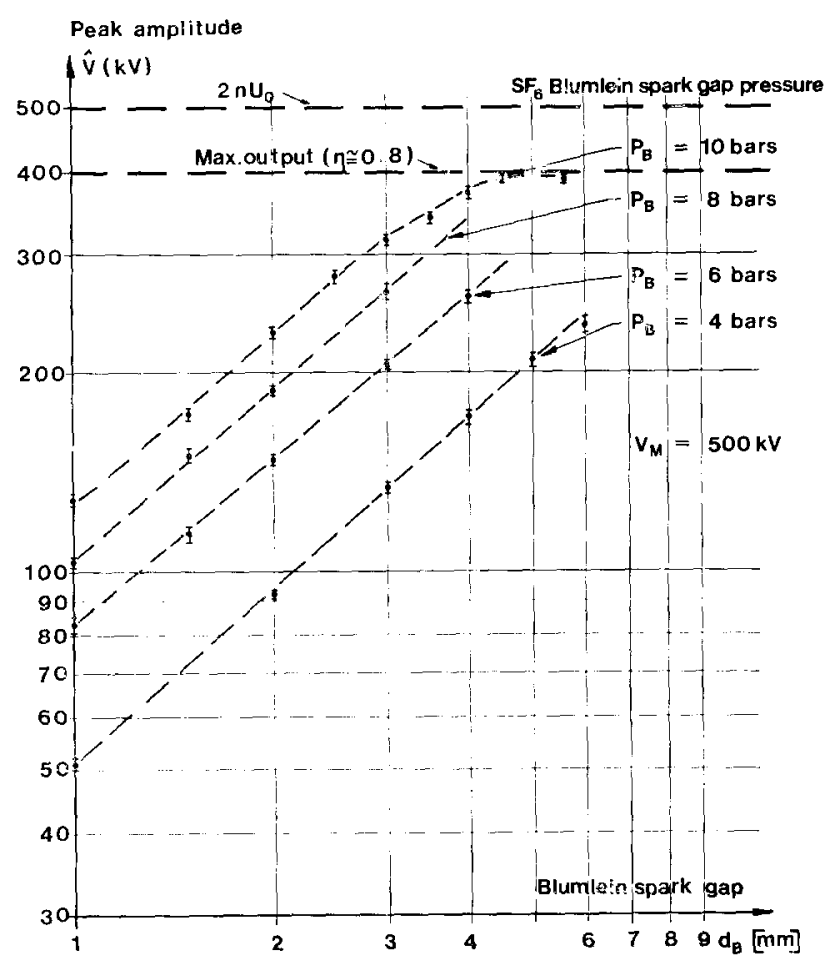

Fig. 6. Peak amplitude of the Blumlein pulse with constant charging voltage $\left(2{ }_{n} U_{0}=500 \mathrm{kV}\right)$ on the $25 \Omega$ chamber.
TABLE 2

Comparison between the 25 and the $40 \Omega$ chamber.

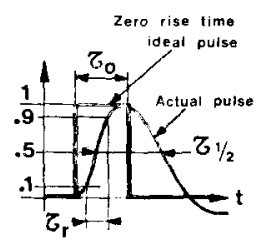

\begin{tabular}{|c|c|c|c|}
\hline Chamber & $25 \Omega$ & \multicolumn{2}{|c|}{$40 \Omega$} \\
\hline$\tau_{0} \quad(n s)$ & 3.1 & 2.8 & 5.5 \\
\hline$\tau_{r} \quad(\mathrm{~ns})$ & $1.5 \pm 0.1$ & $2.2 \pm 0.1$ & $2.1 \pm 0.1$ \\
\hline$\tau_{\frac{1}{2}} \quad(\mathrm{~ns})$ & $3.0 \pm 0.2$ & $4.0 \pm 0.4$ & $6.0 \pm 0.3$ \\
\hline
\end{tabular}

The long pulse has not been used on the large $25 \Omega$ chamber.

gaussian distributions). In fig. 7 a typical distribution of the amplitude of the peak voltage measured on the double gap streamer chamber can be seen.

The rise time of the pulse measured on the chamber is $1.7 \mathrm{~ns}$ for the $25 \Omega$ large chamber and only $2.2 \mathrm{~ns}$ on the small $40 \Omega$ chamber (see fig. 8). The contrary could have been expected (see ref. 1). However it is better on the $25 \Omega$ chamber because the transmission line connecting the coaxial Blumlein to the strip line geometry of the chamber was more carefully matched in impedance for the large chamber. The values of the rise times are given in table 2 . They are corrected for the $0.7 \mathrm{~ns}$ intrinsic rise time of the transient digitiser oscilloscope used (TEKTRONIX R7912+7A19 vertical amplifier). The corresponding widths of the pulse on the chambers are also given.

\section{The streamer chamber}

The tests have been performed in two different chambers:

- a $40 \Omega$ small $9 \times 23 \times 23 \mathrm{~cm}^{3}$ single gap chamber previously described ${ }^{1}$ );

- a $25 \Omega$ large $(2 \times 10) \times 35 \times 35 \mathrm{~cm}^{3}$ double gap chamber (see figs. 9-12).

The large chamber has mainly been built in order to solve the difficult problem of the construction of the high voltage central plane which had to withstand the expected $60-70 \mathrm{kV} / \mathrm{cm}$ electrical stress necessary to produce streamers in hydrogen at atmospheric pressure. But it also was designed as a half-scale model of the chamber we had foreseen building for the physics experimeats. The main features of the design are:

a) The maximum electrical stress outside the plexi- 


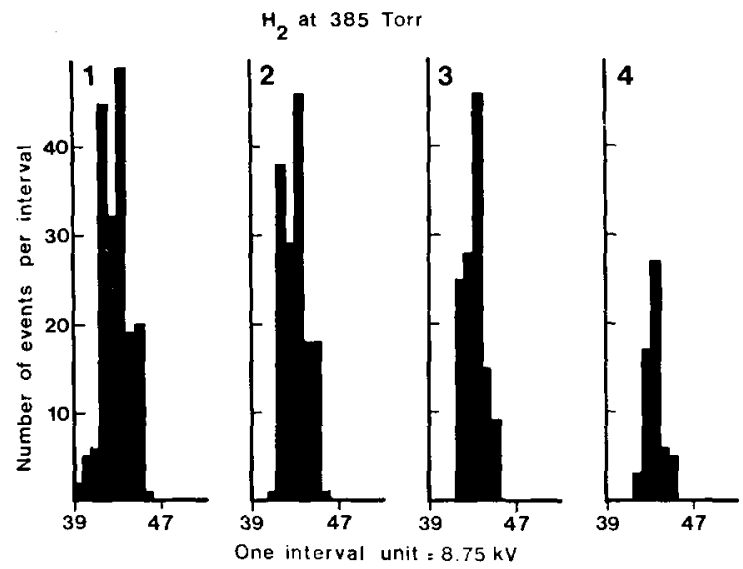

Fig. 7. (a) Typical statistical distribution of the 3 ns peak field pulses on the chamber and of the corresponding pictures with various tracks qualities. $\mathrm{H}_{2}$ at 385 torr. The Blumlein spark gap conditions were $p=10.4$ bar $\left(\mathrm{SF}_{6}\right), \quad$ gap $=5.8 \mathrm{~mm}$, $2 n U_{0}=500 \mathrm{kV}$.

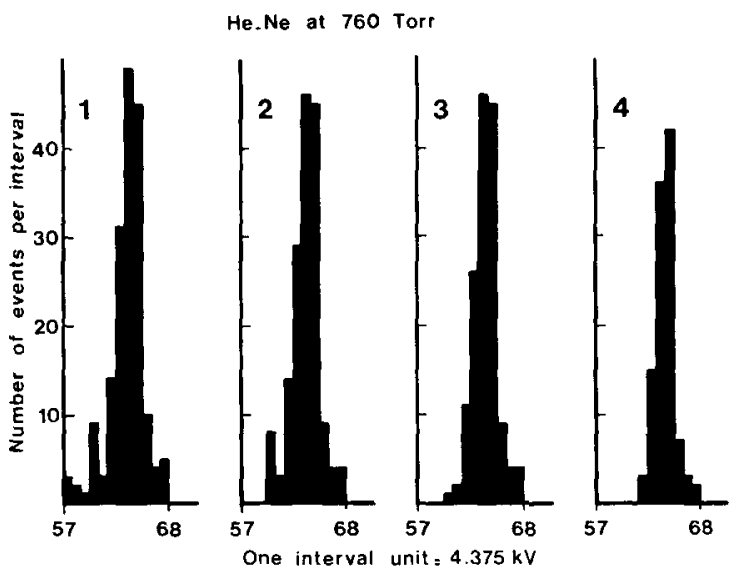

Fig. 7. (b) Typical statistical distribution of the 3 ns peak field pulses on the chamber and of the corresponding pictures with various track qualities. He-Ne at 760 torr. The Blumlein spark gap conditions were: $p=9.4$ bar $\left(\mathrm{SF}_{6}\right), \quad$ gap $=3.6 \mathrm{~mm}$, $2 n U_{0}=340 \mathrm{kV}$.

(a)

Distribution

Pulses

All tracks
Weak good and bright tracks
Histogram number

Number of events

Percentage \%

Mean value $(\mathrm{kV})$

Standard deviation (kV)

Jitter \%

$\begin{array}{cc}1 & 2 \\ 179 & 151 \\ 100 & 84.4 \\ 381.5 \pm 1.0 & 384.1 \pm 1.0 \\ 14.0 \pm 0.7 & 11.4 \pm 0.7 \\ 8.6 & 7.0\end{array}$

$\begin{array}{cc}3 & 4 \\ 123 & 58 \\ 68.7 & 32.4 \\ 383.3 \pm 0.9 & 385.9 \pm 1.1 \\ 9.6 \pm 0.7 & 7.9 \pm 0.8 \\ 5.9 & 4.8\end{array}$

(b)

$\begin{array}{ccc}\text { Distribution } \quad \text { Pulses } & \text { All tracks } & \begin{array}{c}\text { Weak good and } \\ \text { bright tracks }\end{array}\end{array}$

Histogram number

Number of events

Percentage $\%$

Mean value $(\mathrm{kV})$

Standard deviation ( $\mathrm{kV}$ )

Jitter \%

1
176
100
$280 \quad \pm 0.7$
$8.8 \pm 0.5$
7.4

7.4
12

162
$00 \quad 92.0$

3
148
84.1
$281.8 \pm 0.5$
$6.1 \pm 0.3$
5.1

4

108

61.4

$283.1 \pm 0.5$

$4.8 \pm 0.3$

4.0 glass cells is limited to $2.4 \times E_{0}\left[E_{0}=\right.$ field inside the chamber $=\hat{U} \quad$ (peak voltage) $/$ gap $(10 \mathrm{~cm})]$ on the corona tubes.

b) The homogeneity of the field in the sensitive volume of the chamber is better than $1 \%$.

c) The maximum electric field in the transmission line connecting the chamber to the Blumlein is $200 \mathrm{kV} / \mathrm{cm}$ for the maximum working voltage
( $\sim 650 \mathrm{kV}$ for $\mathrm{H}_{2}$ at $\left.1 \mathrm{~atm}\right)$; this maximum stress is compatible with the electrical strength of Freon 114 used as gas insulation around the chamber.

d) The chamber can be evacuated down to $\sim 10^{-4}$ torr. In order to avoid deformation of the cell wall during the pumping, the pressure outside the cells is always kept within 50 torr of the chamber pressure.

e) The ground window is made of thin $\mathrm{Cu}-\mathrm{Be}$ wires 


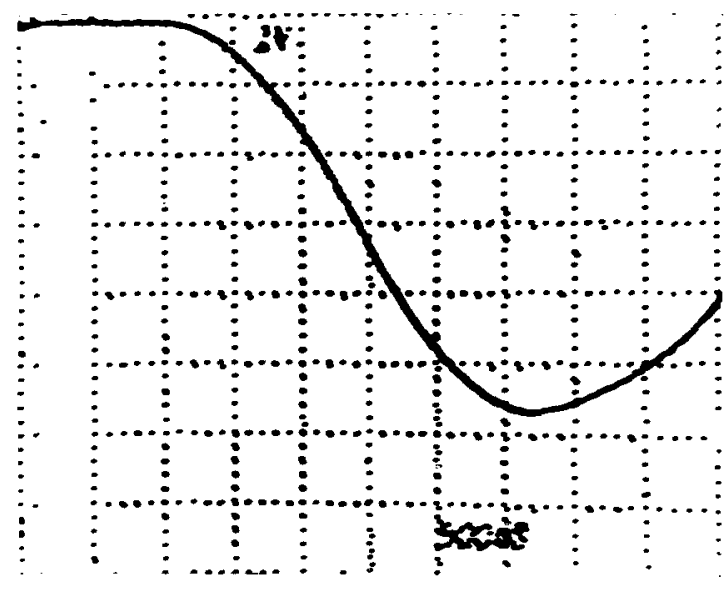

(a)

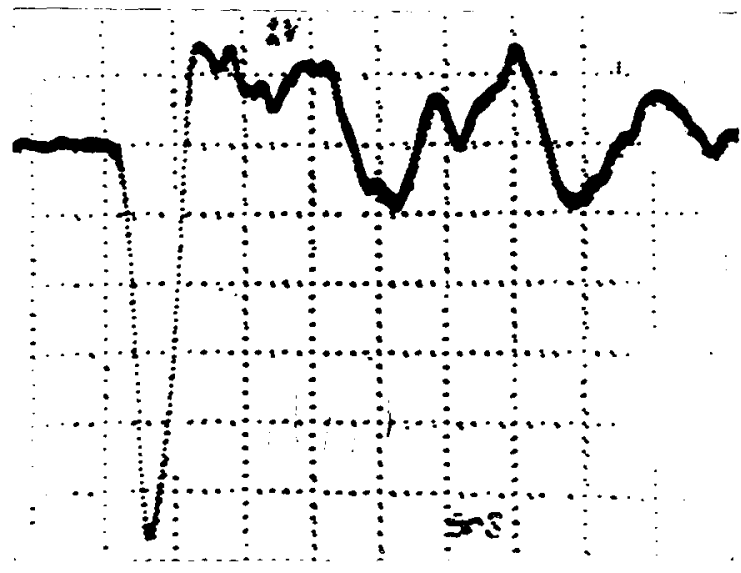

(b)

Fig. 8. High-voltage Blumlein pulse measured on the streamer chamber with $400 \mathrm{kV}\left(2 n U_{0}\right)$ charging voltage on the Marx and $9.3 \mathrm{~atm}$ (abs.) of $\mathrm{SF}_{6}$ and a spark gap of $5.1 \mathrm{~mm}$. (a) $0.5 \mathrm{~ns} / \square$; (b) $5 \mathrm{~ns} / \square$.

( $\varnothing 0.1 \mathrm{~mm}, 2 \mathrm{~mm}$ spacing) glued with lucite between two $2 \mathrm{~mm}$ thick plexiglass foils. This precaution is necessary to avoid breakdown on the wires at the very high fields.

f) The details of the central high voltage plane are given in fig. 10. It must be emphasized that the field at the triple junction between the stainless steel wire, the wall of the cell and the hydrogen gas must be reduced to a minimum in order to prevent severe breakdown. This is why the plexiglass frame is coated with aluminium on the mesh side. Besides, there is good evidence that the smaller the diameter $2 r$ of the mesh wire the better the high voltage performance for a given $r / h$ value ( $h=$ distance between two wires of the mesh). This could be due to the fact that the ratio $r / h$ controls the field on the wires $E_{r}=\sim(r / h) E_{0}$ ( $E_{0}$ is the gap field) and $r$ controls the extent by which the field in the gas is $\gg E_{0}$. Then the gas amplification factor around the wires and thus the number of parasitic streamers increase exponentially with $r$ for a given value of $E_{0}$.

g) The chamber is matched to its $25 \Omega$ characteristic impedance using $4 \times 100 \Omega$ carbon resistors (Silohm type RS3 from Carbone Lorraine France) installed in parallel in the central plane of the chamber.

Two different kind of technical problems were encountered during the first testing period of this chamber:

- gas pollution inside the cell killing the track sensitivity within minutes;

- sparking from the central mesh at $\sim 50 \mathrm{kV} / \mathrm{cm}$ which was too low for getting streamers at atmospheric pressure in hydrogen.
The first problem was quickly solved by improving the control of the load on the main cell gaskets $\left(\sim 30 \mathrm{~kg}^{*} / \mathrm{cm}\right)$ and by using better rubber quality (cast and not stretched). A special designed cylindrical groove had made this problem more important than usual because we tried to minimize the gas pocket in order to prevent sparking in this low dielectric constant $\left(\varepsilon_{1}=\sim 1\right.$ for gas) region, where the electrical stress is multiplied by $\varepsilon_{2} / \varepsilon_{1}=\sim \varepsilon_{2}\left(\varepsilon_{2}=\sim 2.6\right.$ for plexiglass and 4.3 for araldite). The cell was then tight (down to an He vacuum detector sensitivity which is $<10^{-8}$ torr $\left.\cdot 1 / \mathrm{s}\right)$ and track sensitivity could be maintained over many hours even without gas flushing. This also proved that plexiglass, rubber gaskets and gluing material do not give critical outgasing effects.

To overcome the second difficulty the central plane of the streamer chamber was coated with a urethane painting (CRC urethane Isolation - Cellpack) in order to decrease the electric stress on the wires of the mesh by the $\varepsilon$ dielectric constant factor of urethane $(\sim 3.2$ at $1 \mathrm{MHz}$ ). This improved the performance of the chamber and allowed the first good tracks in $\mathrm{H}_{2}$ at atmospheric pressure to be obtained. Before painting the mesh, the effects of the polarity have been investigated. With the central plane negative the sparking threshold is higher by about $10 \%$ than with a positive polarity. This apparent inconsistency comes from the fact that with the positive polarity the cathode side is the window which is not as good as the central mesh because the triple junction between the wal! of the cell, the window and the $\mathrm{H}_{2}$ gas is more stressed than the equivalent region of the central plane (wires instead of 


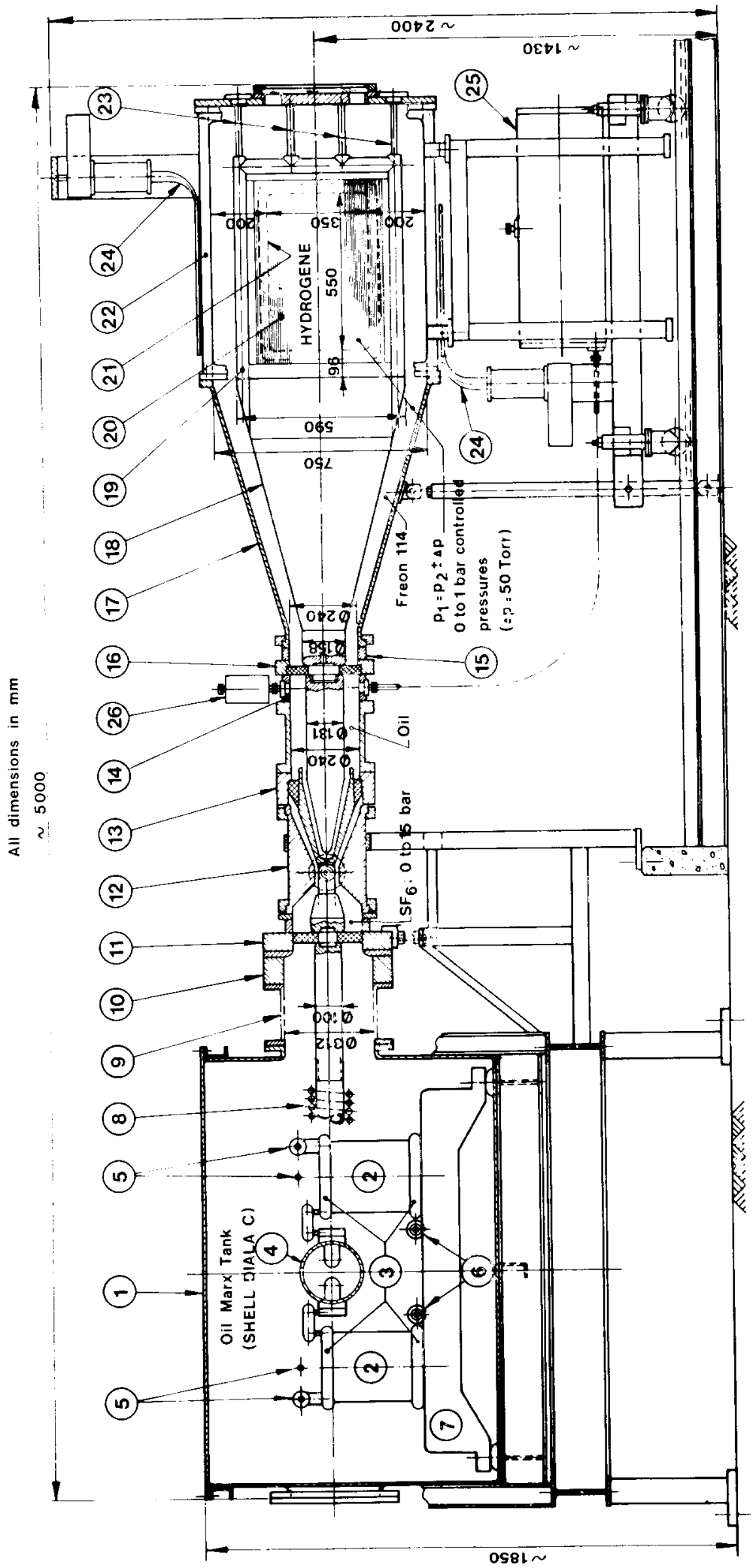




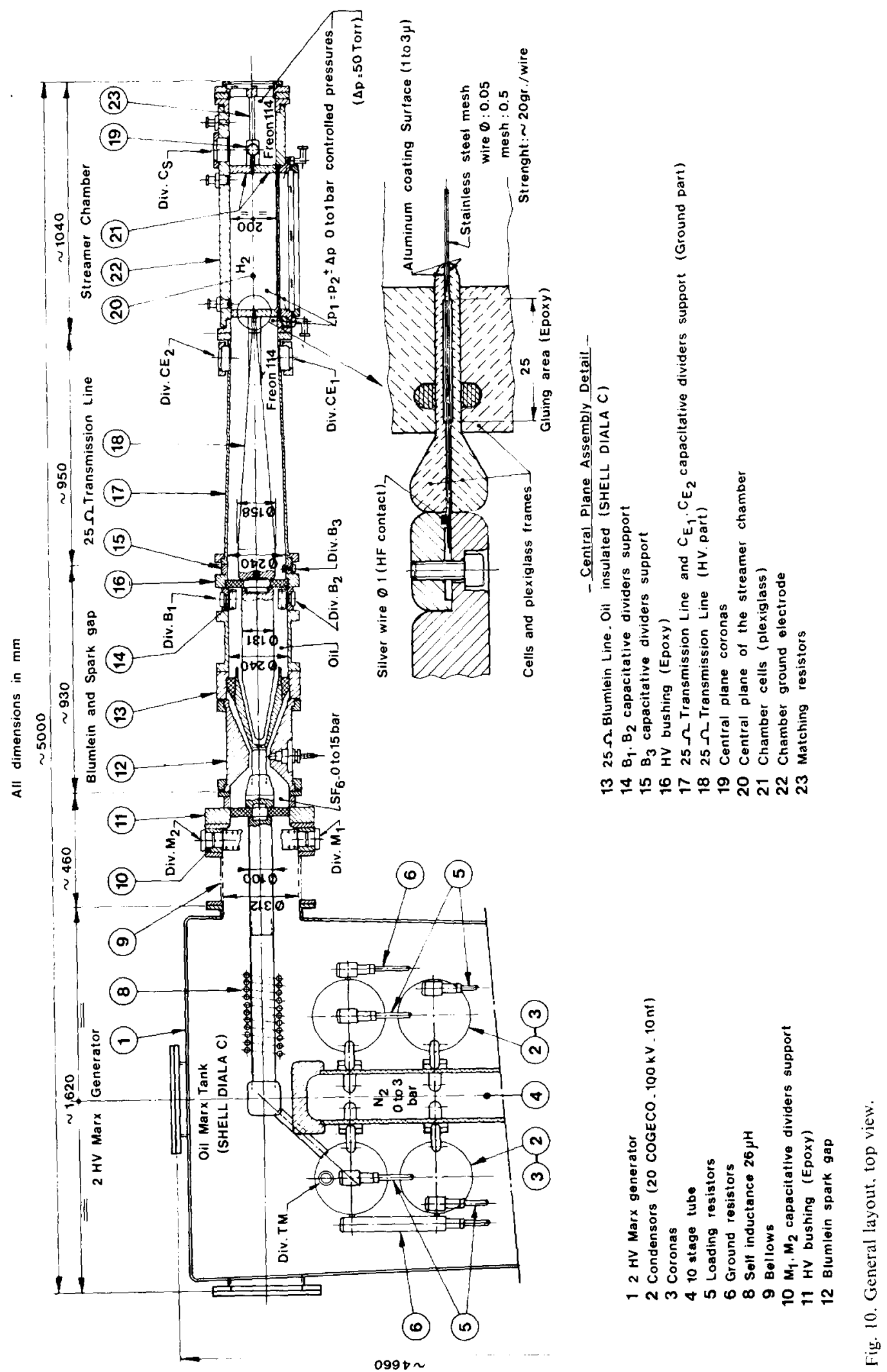




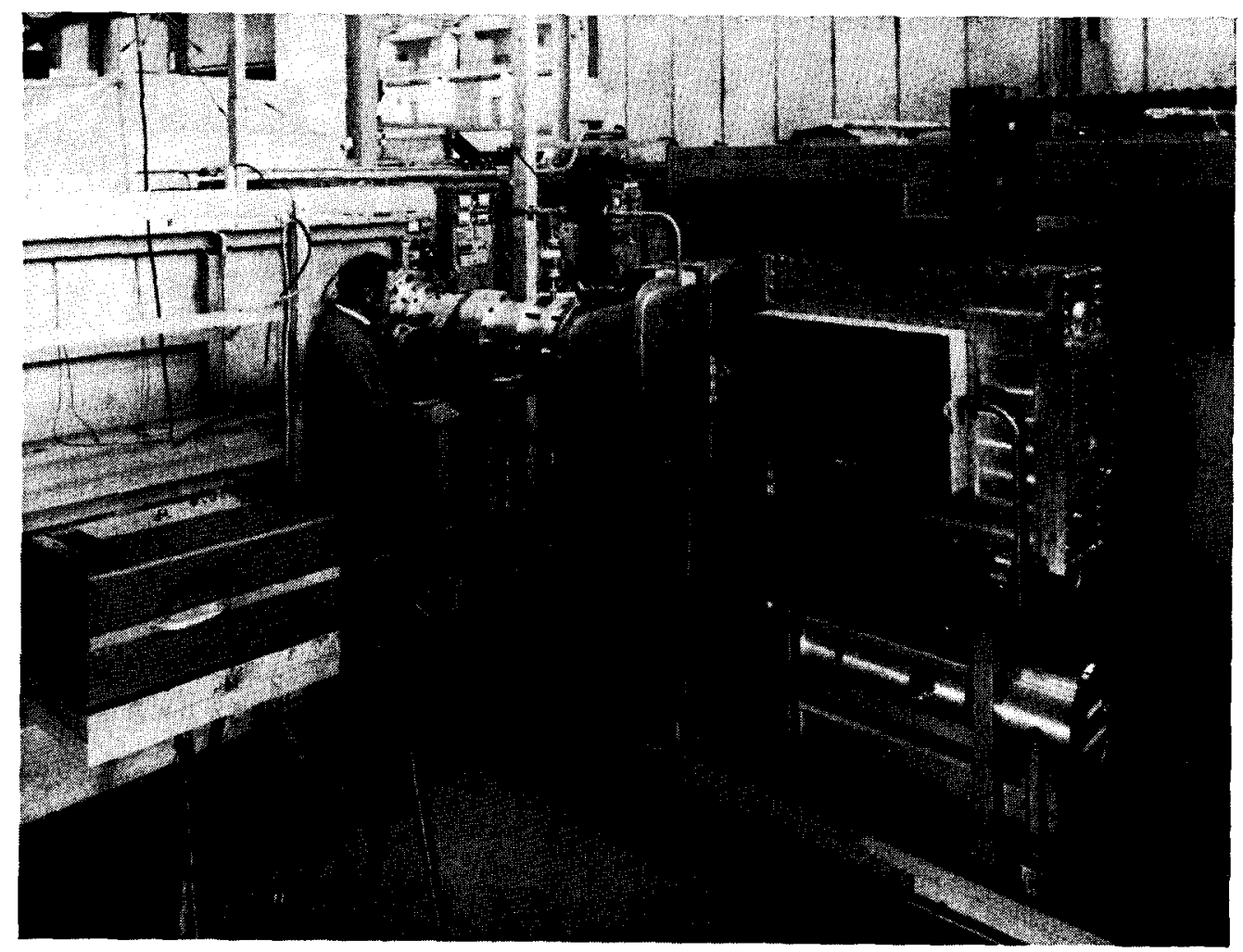

Fig. 11. The $(2 \times 10) \times 35 \times 55 \mathrm{~cm}^{3} \mathrm{H}_{2}$ streamer chamber.

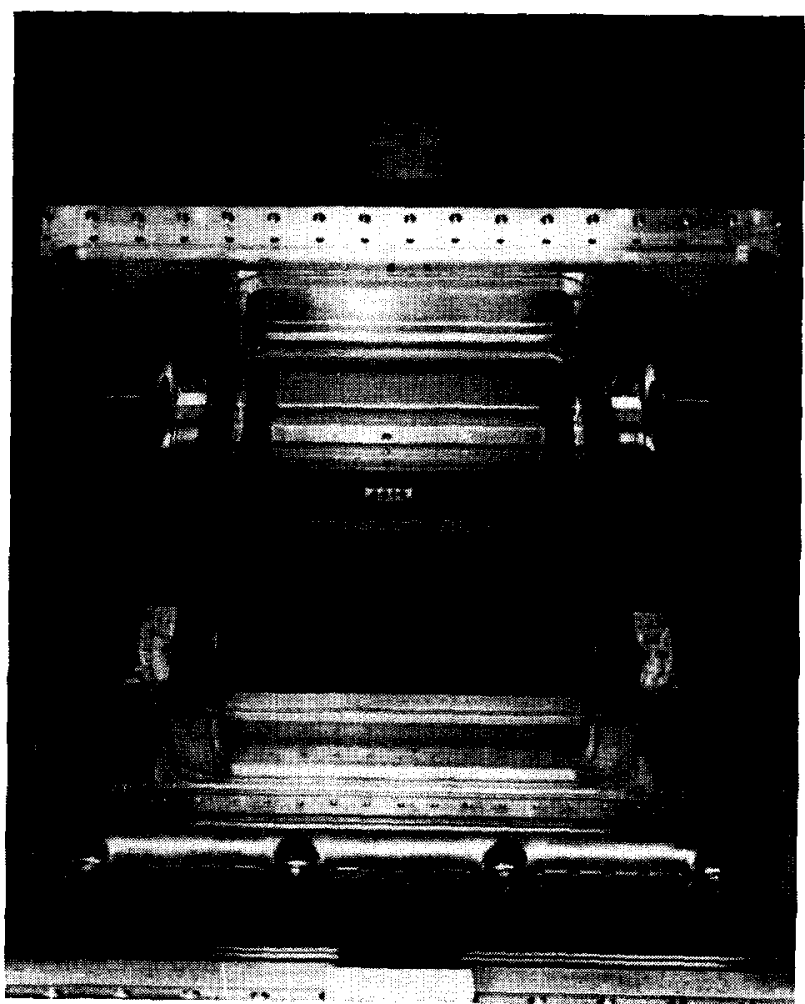

a conducting plane and worse mechanical fitting introducing gas pockets).

The central plane after the urethane painting withstood a stress of $66 \mathrm{kV} / \mathrm{cm}$ without sparking and this with the most severe polarity (negative). The window side did not spark either for this high field value but only with positive polarity. An obvious improvement of the chamber would be to design the window in a similar manner as the design of the central plane.

\section{Photography}

From previous results ${ }^{1}$ ) obtained using direct photography, it was clear that one way of getting better pictures in hydrogen was to use image intensifiers as previously indicated by Falomkin et al. ${ }^{8}$ ).

Most of our results were obtained using a three stages electrostatic image intensifier camera. This camera was built at $\mathrm{CERN}^{9}$ ) using RCA components (RCA $8505 / \mathrm{Vl}$ and $8505 / \mathrm{V} 2$ ). The photocathode diameter is $37 \mathrm{~mm}$. The tube resolution is $\sim 15$ line pairs $/ \mathrm{mm}$ and the gain is $\sim 2 \times 10^{4}$.

Fig. 12. Inside of the streamer chamber. The plexiglass body of the cell, the coronas and the high voltage plane can be seen. 
F. ROHRBACH et al.

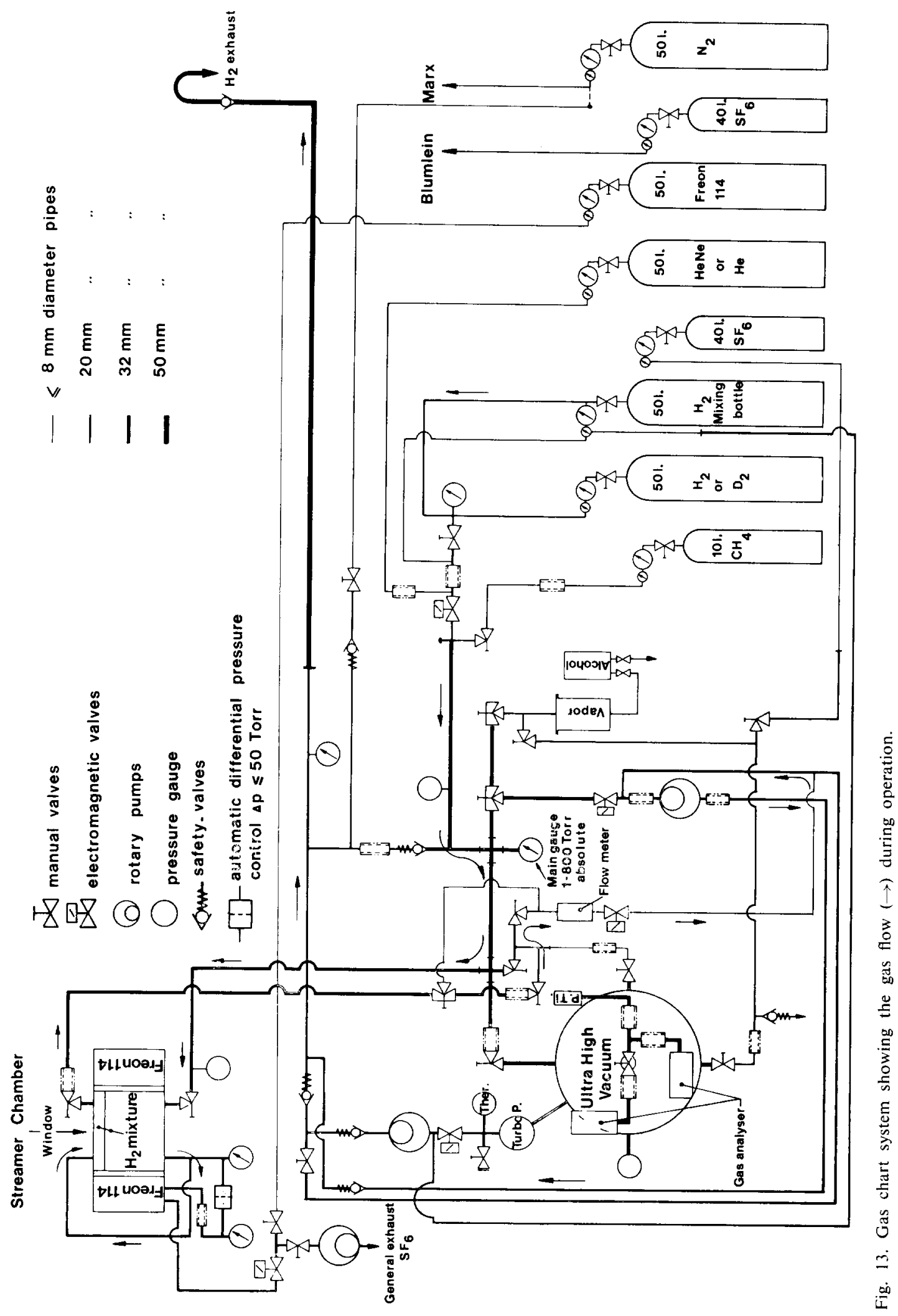


Short tests have also been made with a four stages magnetically focused image intensifier from the EMI company. This tube has a better resolution $(\sim 35$ line pairs $/ \mathrm{mm}$ ) and a larger photocathode diameter $(48 \mathrm{~mm})$. The EMI gain is much larger ( $\sim 50$ times) than the RCA gain. However the output phosphor is not coupled through fiber optics to the film. Thus a classical optical camera system had to be used. This reduced the actual gain of the EMI system which was then only $\sim 3$ times better than the RCA one. This EMI arrangement could only be operated in a continuous mode. This gives rise to severe background light problems and consequently most of the results were obtained with the RCA camera.

The film used for all pictures was SO 143, a special film for streamer chamber developed by Kodak.

The optics we used gave a demagnification factor of 20 . The $F$ stop number was 3.5 for the RCA image intensifier.

\section{Gas system}

After quite a long testing period the mixing gas chart has been built according to fig. 13. The system is more complicated than usual streamer chamber gas chart because of two new difficulties:

- working from vacuum to atmospheric pressure in the chamber;

- using hydrogen gas.

It was also decided to be able to mix up to four

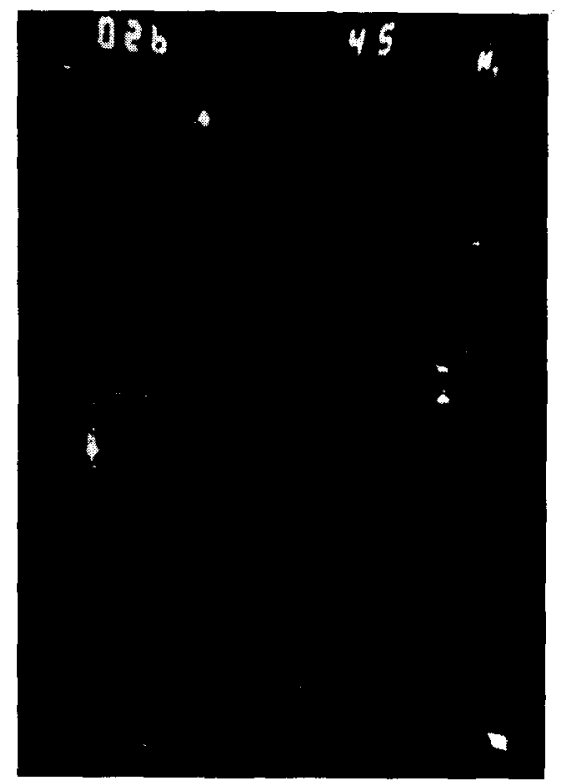

Fig. 14. Typical cosmic ray in the streamer chamber (track length $\sim 35 \mathrm{~cm}) . \mathrm{H}_{2}$ at 725 torr. different gases or vapors. A complete gas analysing system was also available in order to control impurities and gas mixing. A monopole GEC gas analyser has been used for this purpose.

Before using the gas in the chamber, the streamer chamber was always evacuated down to a few $10^{-4}$ torr residual pressure. The mixed gas was prepared before admission to the chamber by isolating the chamber from the gas chart. Usually $15 \mathrm{~atm}$ of gas was prepared and put in the so-called $\mathrm{H}_{2}$ mixing bottle. This amount of gas was enough for a day of operation flushing the gas at the average rate of $0.51 / \mathrm{min}$. At any time the gas flowing in or getting out from the chamber could be analyzed for monitoring its composition.

\section{Results}

The most important result of these experimental studies is that good tracks without sparking have been obtained in $\mathrm{H}_{2}$ and $\mathrm{D}_{2}$ up to the atmospheric pressure (see figs. 14 and 15 compared to 16 and 17 ).

The following results are the main features coming from

6000 pictures obtained in the $9 \mathrm{~cm}$ gap test chamber and

8000 pictures in the $2 \times 10 \mathrm{~cm}$ gap model chamber.

Most of the time cosmic ray tracks were used for triggering the chamber (average counting rate $\sim 1 / 7 \mathrm{~s}$ ). Some tests were made in the small chamber using a $\beta$-source ${ }^{90} \mathrm{Sr}$ of $100 \mu \mathrm{Ci}$ (as in ref. 1).

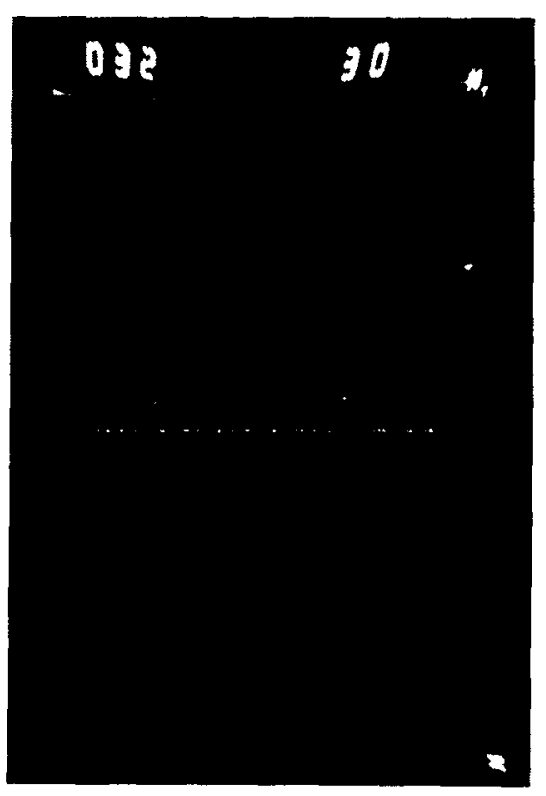

Fig. 15. Typical cosmic ray in the streamer chamber (track length $\sim 35 \mathrm{~cm}) . D_{2}$ at 410 torr. 


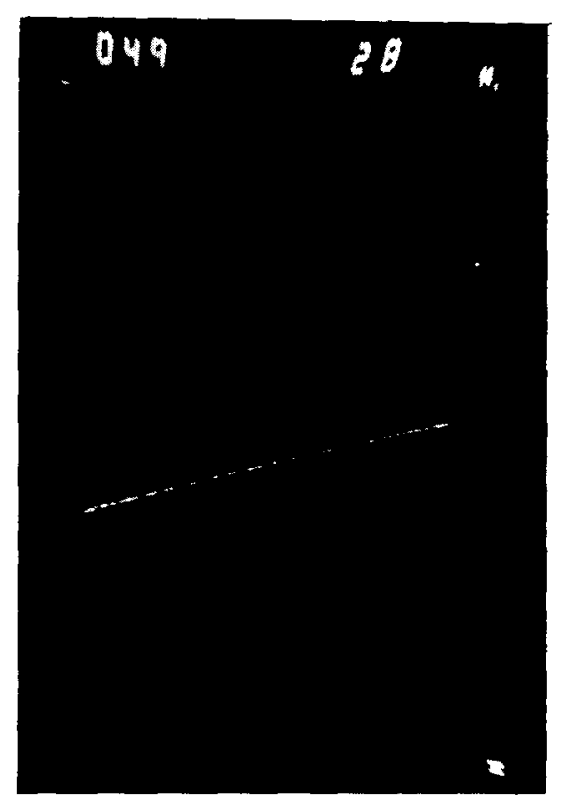

Fig. 16. Typical cosmic ray in the streamer chamber (track length $\sim 35 \mathrm{~cm}) .30 \% \mathrm{He}-70 \% \mathrm{Ne}$ at 760 torr

Most of the pictures were taken using the hydrogen mixture which was found to give the best results $\left(\mathrm{H}_{2}+\frac{1}{2} \% \mathrm{CH}_{4}+\frac{1}{2} \mathrm{ppm} \mathrm{SF}\right)_{6}$, with the aid of the RCA three-stage image intensifier camera with a $\times 20$ demagnification and the SO143 Kodak film. The gas was usually flushed at a rate of $0.5 \mathrm{l} / \mathrm{min}$. For a typical run 180 pictures were usually taken.

\subsection{VOLTAGE-PRESSURE THRESHOLD CONDITIONS}

For each picture the peak voltage of the pulse on the chamber was measured using the Tektronix transient digitizer R7912. The peak value was obtained with a $1-2 \%$ reading error (the instrument itself is given for $\frac{1}{2} \%$ if used with an on-line computer). The pictures were then scanned for track quality, recording special observations and measuring the streamer density for the tracks of even good quality and geometry. The results were then put on data cards for statistical analysis and particularly to correlate peak voltage to track quality. This way of analysing the results allows one to separate the effect of the jitter of the peak field coming mainly from the Blumlein spark gap from the statistical fluctuations of the streamer formation. Therefore the relative values of the peak field are known with a good accuracy (down to $1 \%$ for the largest samples). However, due to calibration uncertainty, the absolute values are known only within about $5 \%$.

As already shown ${ }^{1}$ ) only the peak value of the

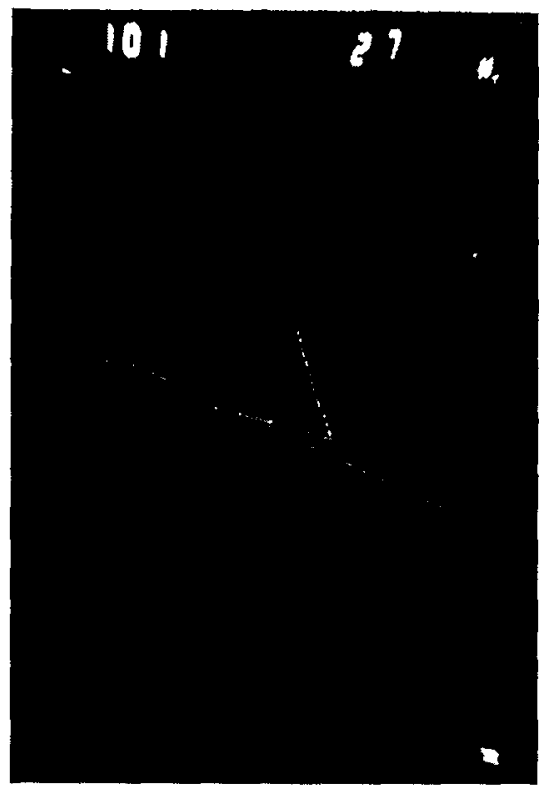

Fig. 17. Typical cosmic ray in the streamer chamber (track length $\sim 35 \mathrm{~cm}$ ). He at 760 torr.

electric field $\hat{E}$ determines the onset of the streamer for a given pulse shape and gas pressure value. The experimental results are in reasonable agreement with the criterion $\hat{E}^{3} \tau_{1 / 2} p^{-2}=$ constant (gas) (see fig. 18). We have found that it is more precise to use for $\tau$ the fwhm $\tau_{1 / 2}$ and not the width at the base of the

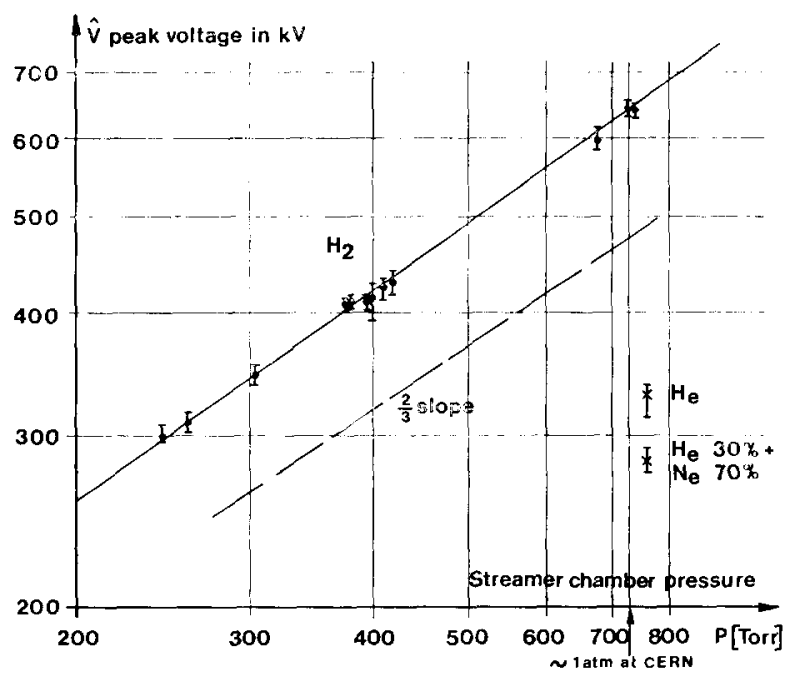

Fig. 18. Dependence of the peak voltage on the $\mathrm{H}_{2}$ pressure for good tracks in the chamber. The limits shown are for bright and weak streamers. The pulse was $3 \mathrm{~ns}$ fwhm and the $\mathrm{H}_{2}$ was mixed with $1 \% \mathrm{CH}_{4}$ and an average of $\frac{1}{2} \mathrm{ppm} \mathrm{SF}_{6}$ giving a memory time of $\sim 2 \mu \mathrm{s}$. The chamber was the $2 \times 10 \mathrm{~cm}$ gap which means that the peak field was $\hat{V} / 10 \mathrm{kV} / \mathrm{cm}$. 
TABle 3

Peak field values for gas at standard atmospheric pressure (760 torr) and temperature $(\sim 20 \mathrm{C})$ and for a $3 \mathrm{~ns}$ fwhm.

\begin{tabular}{ccc}
\hline Gas & $\hat{E}$ relative & $\hat{E}(\mathrm{kV} / \mathrm{cm})$ \\
\hline $30 \% \mathrm{He}-70 \% \mathrm{Ne}$ & 1 & $28 \pm 2$ \\
$\mathrm{He}$ & $1.17 \pm 0.01$ & $33 \pm 2$ \\
$\mathrm{H}_{2}$ (and $\left.\mathrm{D}_{2}\right)$ & $2.34 \pm 0.02$ & $66 \pm 3$ \\
\hline
\end{tabular}

pulse ${ }^{1,5}$ ). The value of $\hat{E}$ is determined by calculating the mean of the peak values from a sample of pulses corresponding to the same track quality for a given set of experimental conditions. With the 3 ns fwhm pulse and taking only the tracks with the same constant good quality and position in the streamer chamber (in the middle of the chamber) we have obtained the results given in table 3 .

The peak field values for $D_{2}$ are $1-2 \%$ lower than for $\mathrm{H}_{2}$ but this effect lies within the experimental errors. The sensitivity of the track brightness to the peak field value has also been measured with a good accuracy. For this purpose the tracks quality has been compared by considering only the tracks in the central part of the chamber. The results are in agreement with the previous measurements obtained with direct photography ${ }^{1}$ ): at atmospheric pressure hydrogen is about two times more sensitive to voltage fluctuations than $\mathrm{He}-\mathrm{Ne}$.

Typical results are shown in table 4.

It is interesting to point out that at low pressure $\mathrm{H}_{2}$ is much less sensitive to voltage fluctuations. This could be understood because for a given pulse length the variation of the brightness $B(\perp \boldsymbol{E})$ with the peak field at the onset of the streamer is almost linear with $p$ :
$S_{B}=\frac{\mathrm{d} B(\perp E)}{\mathrm{d} E} \propto p^{5 / 6}$, (estimated from ref. 2$)$

Using the relationship $\hat{E}^{3} \tau_{p}^{-2}=$ const., one can express $S_{\mathrm{B}}$ as a function of the peak field:

$S_{B} \propto \hat{E}^{5 / 4}, \quad$ or $\quad \Delta B(\perp E) \propto \hat{E}^{9 / 4} \Delta \hat{E} / \hat{E}$.

This shows that for a given value of the jitter the fluctuations of the brightness will be more important when the peak field is high.

Experimentally one finds that the efficiency of a streamer chamber is very sensitive to the pulse amplitude jitter because of the great sensitivity of the brightness of the tracks to these voltage fluctuations. The chamber efficiency $\eta$ is defined as the percentage of tracks which can be accepted for a given criterion of quality over the total number of expected pictures.

A simple mathematical model which could describe these effects can be developed. Let us assume that:

a) The pulse height is Gaussian distributed around $\bar{E}$ with a standard deviation $\sigma_{\bar{E}}$.

b) The probability of getting a given quality of track for a given pulse height $\hat{E}$ is $P(\hat{E})=\exp \left[-(\hat{E}-I)^{2} / 2 \sigma_{I}^{2}\right]$, where $I$ is the ideal voltage for getting the corresponding quality and $\sigma_{I}$ describes its sensitivity to voltage fluctuations.

With these two hypotheses it is possible to deduce that the average efficiency $\eta$ is

$\eta=\frac{\bar{\sigma}}{\sigma_{\bar{E}}} \exp \left\{-\frac{(\bar{E}-I)^{2}}{2\left(\sigma_{\bar{E}}^{2}+\sigma_{I}^{2}\right)}\right\}$,

with

$\bar{\sigma}=\frac{\sigma_{E} \sigma_{I}}{\sqrt{\left(\sigma_{\tilde{E}}^{2}+\sigma_{I}^{2}\right)}}$.

The observed distribution for the tracks of a given

TABle 4

Effect of amplitude jitter on the brightness of the tracks.

\begin{tabular}{|c|c|c|c|c|c|c|c|}
\hline$\underset{\text { (torr) }}{p}$ & Gas & $\begin{array}{c}\text { Pulse } \\
\text { jitter } \\
(\%)\end{array}$ & \multicolumn{3}{|c|}{$\hat{E}\rangle(\sigma(\hat{E}))$ in arbitrary units } & \multicolumn{2}{|c|}{$2.35 \sigma(\hat{E}) / \hat{E}$} \\
\hline 760 & $\mathrm{He}$ & 6.9 & $3.59(0.07)$ & $3.78(0.08)$ & $3.87(0.04)$ & 4.6 & 5.9 \\
\hline 385 & $\mathrm{H}_{2}$ & 8.5 & $4.28(0.07)$ & $4.38(0.07)$ & $4.54(0.06)$ & 4.0 & 6.1 \\
\hline
\end{tabular}


TABLE 5

Pulse amplitude mean and standard deviation together with the efficiency measured and calculated for various streamer chamber gas and pressure conditions with a 3 ns fwhm pulse.

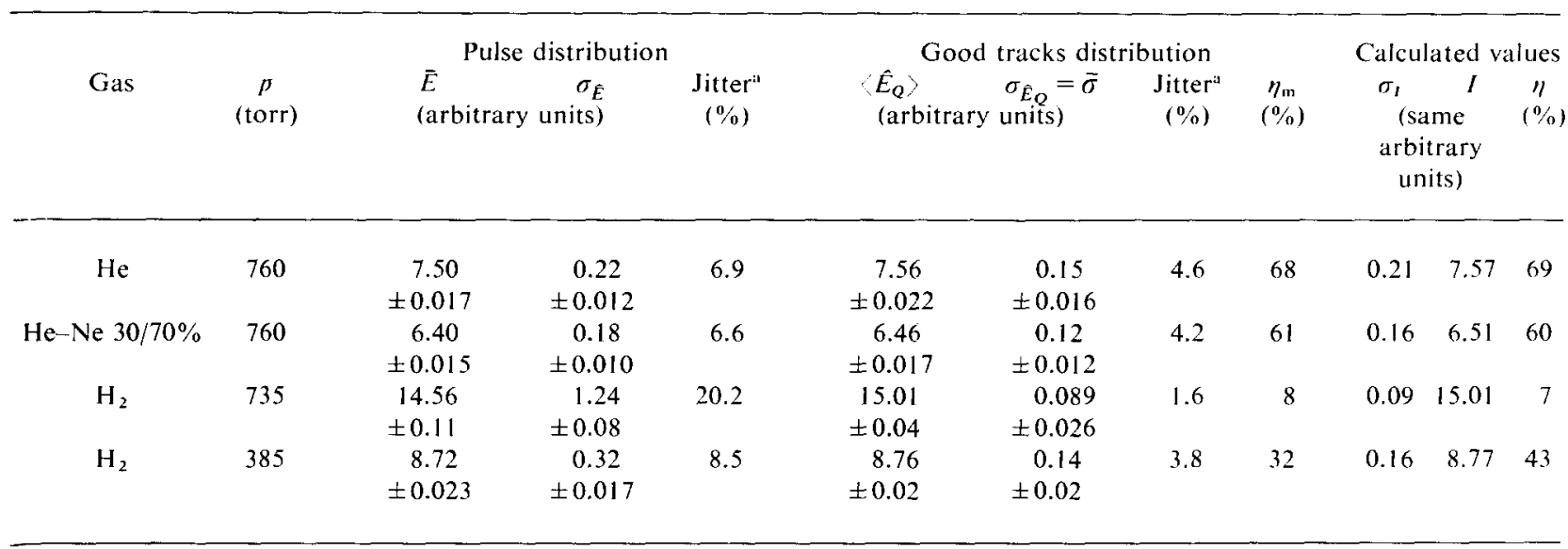

a The jitter is defined as $2.35 \sigma /\langle E\rangle$ in $\%$; this is equivalent to the fwhm for a Gaussian distribution.

quality has a mean which is

$\left\langle\hat{E}_{Q}\right\rangle=\bar{\sigma}^{2}\left(\frac{\bar{E}}{\sigma_{\hat{E}}^{2}}+\frac{I}{\sigma_{I}^{2}}\right)$,

and a standard deviation

$\sigma_{\bar{E}_{Q}}=\bar{\sigma}$.

For a set of experimental conditions, taking a typical run of $\sim 180$ pictures, $\bar{E}$ and $\sigma_{\hat{E}}$ are estimated from the sample mean and standard deviation. Then, selecting only the peak field values of the pictures with a constant quality $Q$ (for example the "good tracks" which means the tracks with no visible fluctuations in brightness and well contrasted small streamers), $\left\langle\hat{E}_{\mathbf{Q}}\right\rangle$ and $\sigma_{\hat{E}_{\mathbf{Q}}}$ are also estimated from this "good" sample. If the pulse distribution is reasonably Gaussianlike then the corresponding $\sigma_{I}, I$ and $\eta$ values can be calculated from eqs. (1)-(4):

$\sigma_{I}=\frac{\bar{\sigma} \sigma_{\hat{E}}}{\left.\sqrt{\left(\sigma_{E}^{2}\right.}-\bar{\sigma}^{2}\right)}$,

$I=\sigma_{I}^{2}\left(\frac{\left\langle\hat{E}_{Q}\right\rangle}{\bar{\sigma}^{2}}-\frac{\bar{E}}{\sigma_{\bar{E}}^{2}}\right)$,

and $\eta$ given by eq. (1).

The value of $\eta$ can also be measured. Let us call it $\eta_{\mathrm{m}}$. The two efficiencies are compared. The agreement seems quite good (see table 5) and enough for giving the very interesting possibility of estimating the efficiency when the pulse jitter is known. When the pulse is correctly centered on the average value $I$ for ob- taining the best track quality then $\bar{E}=I$ and the efficiency is maximum $\eta_{\max }=\sigma / \sigma_{\bar{E}}$.

If some fluctuation of the brightness of the tracks is accepted (from weak to bright rejecting the very weak and the very bright which are not acceptable in a high energy physics experiment) then the efficiency is better, as shown in table 6 .

At atmospheric pressure in $\mathrm{H}_{2}$ the jitter of the Blumlein was quite large because the high voltage had to be raised to the high voltage limits of the spark gap of the Blumlein.

From all these results it can be estimated that for $\mathrm{H}_{2}$ or $\mathrm{D}_{2} 90 \%$ efficiency for track measurement could be achieved with a maximum amplitude jitter of $2-3 \%$ [estimated from tables 5 and 6 and eqs .(1) and (2), which gives $2.35 \sigma_{\hat{E}} /\langle\hat{E}\rangle=2.35 \sigma_{I} \sqrt{\left(1-\eta^{2}\right)} /\langle\hat{E}\rangle$ $=2.3 \%]$. With the usual $4-5 \%$ fwhm pulse ${ }^{10}$ ) the efficiency in $\mathrm{H}_{2}$ can be estimated as from 79 to $72 \%$.

\subsection{Size, Shape and Luminosity OF the Streamers}

The main characteristic of a track photographed using an image intensifier instead of a direct camera is the better homogeneity of the dimensions of the streamers created along the particle trajectory (see figs. 14-17). This is likely due to the fact that the high light gain obtained with the I.I. $\left(\sim 2 \times 10^{4}\right.$ with the 3 stages RCA and $\sim 6 \times 10^{4}$ with the 4 stages EMI) allows photographs of the head of the avalanche before the onset of the streamer to be taken. As the luminous front of a streamer is moving about ten times faster ${ }^{1.12}$ ) than the head of the avalanche before the onset of the streamer development, the 


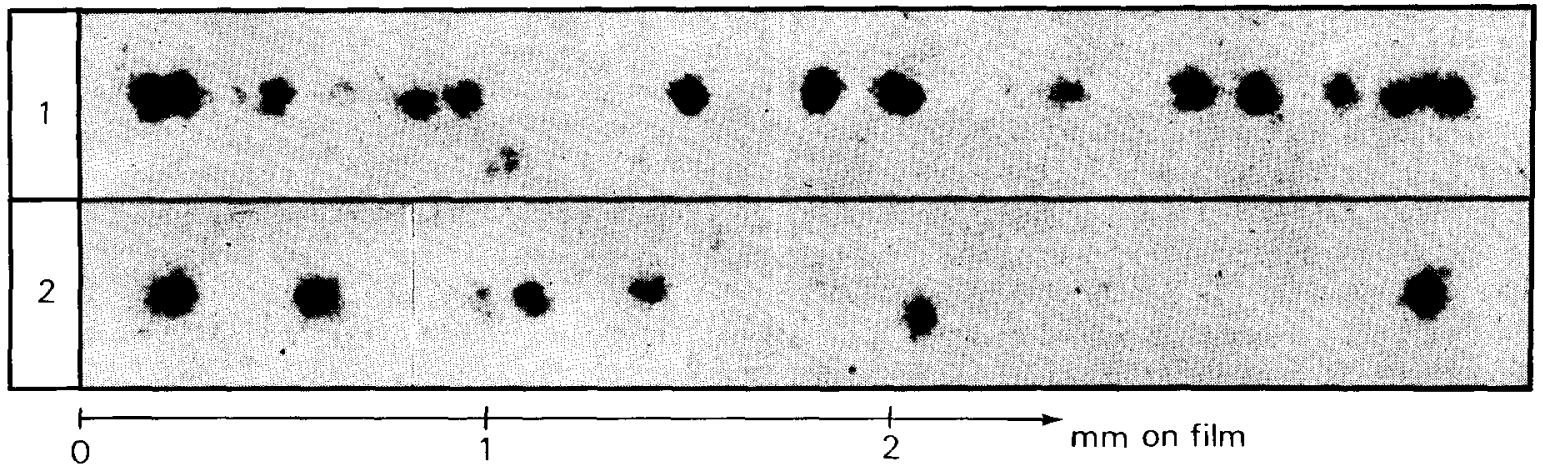

Fig. 19. Size of the image of the streamers in $\mathrm{H}_{2}$ with two different demagnifications: (1) Demagnification $\times 20 . \mathrm{H}_{2}(725$ torr). (2) Demagnification $\times 10, \mathrm{H}_{2}$ (510 torr).

TABle 6

Standard deviation and efficiencies when considering weak good and bright tracks. Same conditions as for table 5.

\begin{tabular}{|c|c|c|c|c|c|c|c|c|c|}
\hline & Gas & $\sigma_{\hat{E}}$ & $\begin{array}{l}\text { Jitter } \\
(\%)\end{array}$ & $\bar{\sigma}$ & $\begin{array}{c}\text { Jitter } \\
(\%)\end{array}$ & $\begin{array}{r}\eta_{m} \\
(\%)\end{array}$ & $\sigma_{I}$ & $\begin{array}{c}\text { Jitter } \\
(\%)\end{array}$ & $\begin{array}{c}\eta \\
(\%)\end{array}$ \\
\hline $\mathrm{He}$ & 760 torr & $0.22 \pm 0.012$ & 6.9 & $0.19 \pm 0.011$ & 5.9 & 88 & 0.36 & 11.2 & 85 \\
\hline $\mathrm{H}_{2}$ & 735 torr & $1.24 \pm 0.08$ & 20.2 & $0.32+0.035$ & 5.0 & 29 & 0.33 & 5.2 & 24 \\
\hline $\mathrm{H}_{2}$ & 385 torr & $0.32 \pm 0.017$ & 8.5 & $0.22 \pm 0.014$ & 6.1 & 69 & 0.30 & 8.0 & 68 \\
\hline
\end{tabular}

statistical distribution of the brightness of the dots in the avalanche mode shows a smaller spread. The quality of tracks using an image intensifier is thus greatly increased.

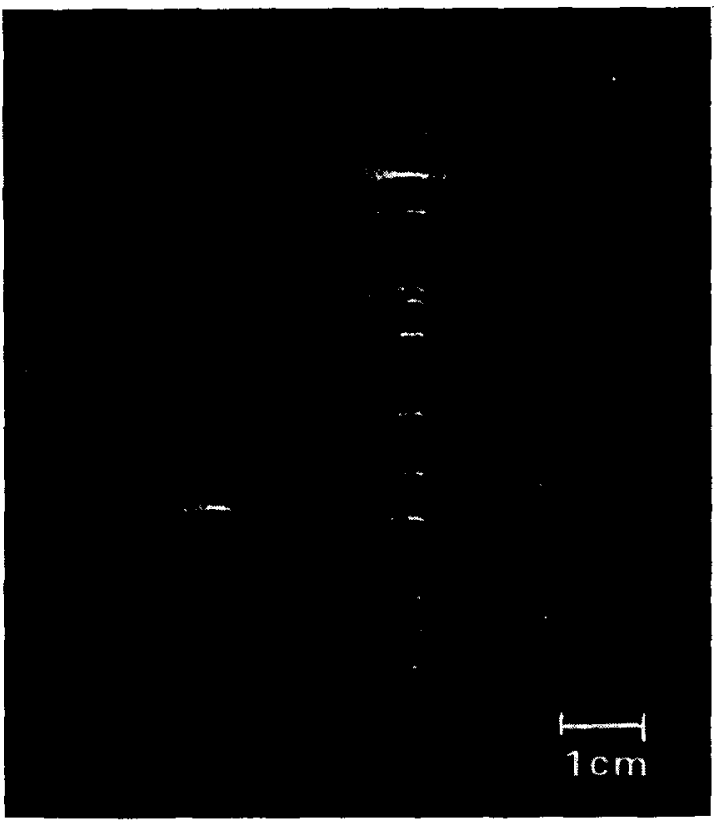

Fig. 20. Track in $\mathrm{H}_{2}$ photographed along the field lines 90 to $\boldsymbol{E}$ in the $9 \mathrm{~cm}$ chamber at 500 torr; $40 \Omega$ chamber.
We have found that the size of the image of the streamers in $\mathrm{H}_{2}$ does not depend on the demagnification $G$, for $G$ varying from 10 to 20 . The streamer sizes lie between 50 and $100 \mu \mathrm{m}$ on the film (see fig. 19). This indicates that the optical system is limited to a resolution of $\sim 10$ line pairs per $\mathrm{mm}$. Consequently the size of the diarreter $\varnothing_{\mathrm{s}}$ of the streamers in space is not known but only the higher limits $\varnothing_{\mathrm{s}}<\sim 500 \mu \mathrm{m}$. The corresponding length of the streamers along the field could not be measured simultaneously because only one image intensi ier was available. However the length of the streamers has been measured separately by putting the RCA image intensifier at $90^{\circ}$ looking through the longitudinal window along the field lines in the $9 \mathrm{~cm}$ gap chamber and at $18^{\circ}$ stereo looking through the main window perpendicular to the field lines in the $2 \times 10 \mathrm{~cm}$ gap chamber (see figs. 20 and 21 ). The length of the streamers in $\mathrm{H}_{2}$ has been found to be from 3 to $8 \mathrm{~mm}$.

Using a conventional measuring table for bubble chamber pictures a regression linear fit has been made on a few straight cosmic ray tracks. The average residual dispersion found is $\sim 300 \mu \mathrm{m}$ which is the value expected for the diffusion of the electrons during the $1 \mu$ s delay between the passage of the cosmic particle and the application of the high voltage on the chamber. The setting error in the presence of a magnetic 
field will not be linited by this effect [the diffusion coefficient is reduced by more than an order of magnitude from 0 to $\left.12 \mathrm{kG}^{13}\right)$ ].

It is possible to photograph simultaneously minimum ionising particles and strongly ionising particles (see figs. 22, 23a and 23b). This will be an advantage for looking at low momentum recoils in hydrogen.

There is no difference in the brightness of the streamers for a track crossing the central plane.

Serious distortions are observed when using an image intensifier. However it has been shown ${ }^{9}$ ) that a precise reconstruction is possible because the distorted image is sufficiently stable (average displacement $\sim 2$ to $3 \mu \mathrm{m}$ on the film over hcurs).

\subsection{EFFECT OF MIXING OTHER GASES AND MEASUREMENT OF THE MEMORY}

Pure hydrogen alone is not convenient because of high background light. The photo-electrons produced on the electrodes by the strong UV emission of the streamers give rise to secondary streamers outside the path of the high energy particle crossing the chamber (see fig. 24). In order to be sure that the effect of

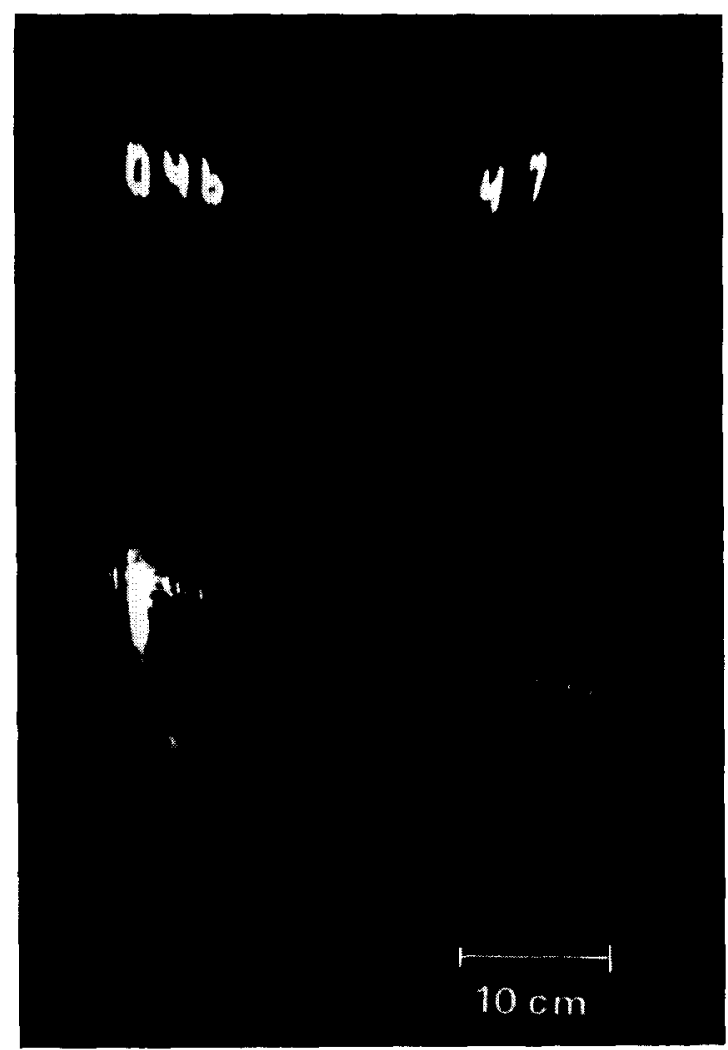

Fig. 21. Track in $\mathrm{H}_{2}$ photographed at $18^{\circ}$ to $E$ in the $2 \times 10 \mathrm{~cm}$ chamber at 375 torr; $25 \Omega$ chamber. impurities was reduced to a minimum we have used the purest $\mathrm{H}_{2}$ available (quality $\mathrm{N} 57$ from Air Liquide France) which is guaranteed for $99.9997 \% \mathrm{H}_{2}$ with $\mathrm{H}_{2} \mathrm{O}<5 \mathrm{vpm}\left(\mathrm{vpm}=\right.$ volume per $\left.10^{6}\right) \mathrm{N}_{2}$ and $\mathrm{O}_{2}<0.5$ vpm.

In order to absorb the UV emitted mainly in the band $1000-1200 \AA$ we have tried various kinds of gases in different proportions. If a visible avalanche is said to emit $\sim 10^{8}$ photons, the absorption coefficient of the gas mixture $\eta$ must be high in order to avoid large photoelectric emission. The UV quantum efficiency of our mesh cathode is not known but it might be of the order of 0.1 maximum. The UV attenuation factor must then be of the order of $10^{8}$ over a few $\mathrm{cm}$ to quench the UV secondary effects. This means that $\mu$ must be of the order of $5 \mathrm{~cm}^{-1}$. On the other hand the impurity content must be small enough to keep the interactions in $\mathrm{H}_{2}$ for a high energy physics experiment close to $100 \% . \mathrm{CH}_{4}$ has been found the best impurity from both aspects; it has a very high photo-absorption cross section in the $1000-1200 \AA$ range $\left(\sigma \sim 20-30 \mathrm{Mb}\right.$ which gives a $\mu$ of 500 to $800 \mathrm{~cm}^{-1}$ at NPT) and the collision cross section on $\mathrm{C}$ is only $\sim 5$ times the collision cross section on $\mathrm{H}$. Thus $0.5 \% \mathrm{CH}_{4}$ in $\mathrm{H}_{2}$ will induce only $\sim 1.3 \%$ interactions on C. Using $0.5 \%$ (in molecules) of $\mathrm{CH}_{4}, \mu$ is $\sim 4 \mathrm{~cm}^{-1}$ for a mixture at atmospheric pressure. The effect is spectacular as can be seen on fig. 25. $\mathrm{H}_{2} \mathrm{O}, \mathrm{H}_{2} \mathrm{~S}$, isobutane, alcohol $\left(\mathrm{C}_{2} \mathrm{H}_{6} \mathrm{O}\right)$ and methylal $\left(\left(\mathrm{CH}_{3} \mathrm{O}\right)_{2} \mathrm{CH}_{2}\right)$ have also been tested but the results were always less

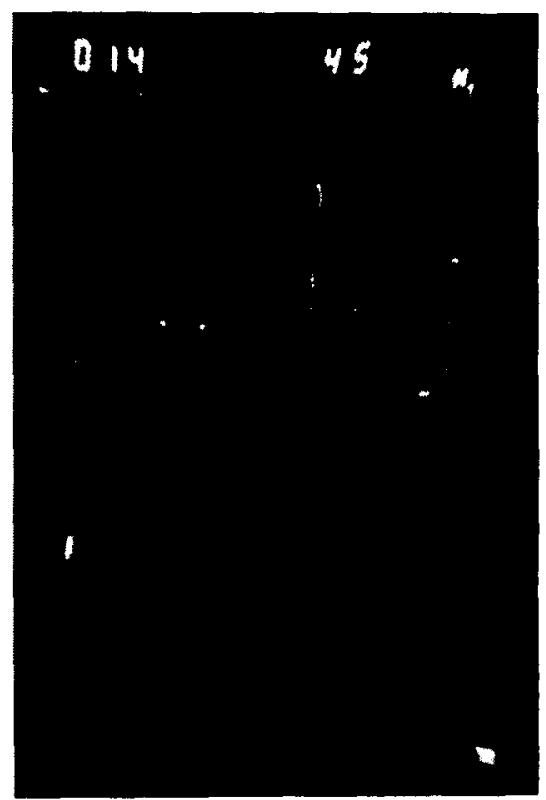

Fig. 22. $\delta$-ray in $\mathrm{H}_{2}$ at 735 torr; $25 \Omega$ chamber. 
interesting. For example, with $\mathrm{H}_{2} \mathrm{O}$ and methylal the brightness of the streamers is strongly decreased to such a point that the central luminous part of the streamers disappears.

In an experiment it is very important in order to take

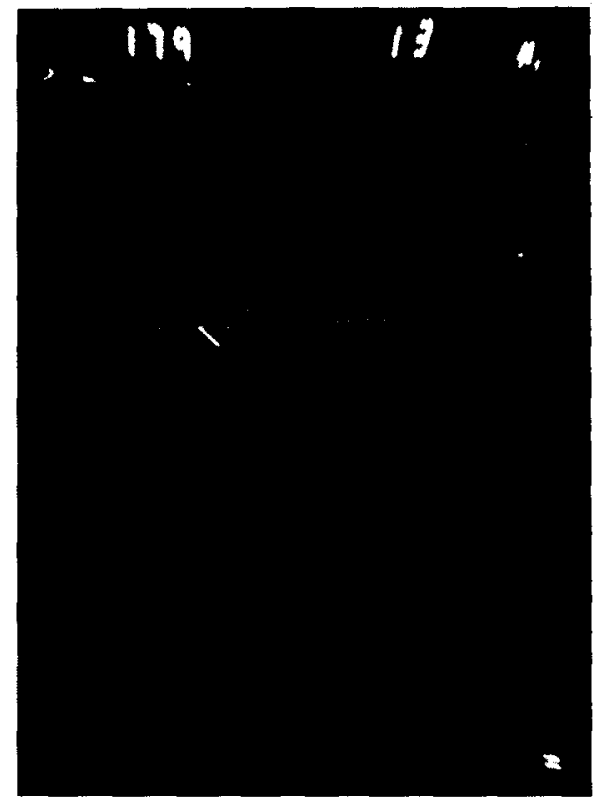

(a)

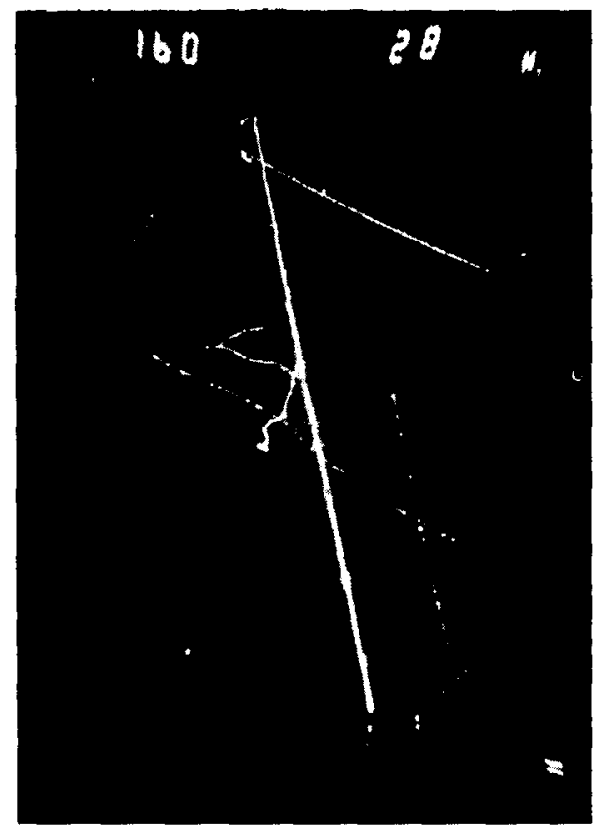

(b)

Fig. 23. (a) Recoil proton or $\delta$-ray in $\mathrm{H}_{2}$ at 410 torr. (b) Recoil of nuclear fragement in $\mathrm{He}-\mathrm{Ne}$ at 760 torr, $25 \Omega$ chamber, window $35 \times 55 \mathrm{~cm}^{2}$. full advantage of the triggering property of a streamer chamber to adjust the memory time of the gas and to keep it constant over long runs (days). The smaller the memory time, the higher the beam flux which can be accepted. The minimum time is determined by the delay necessary for the logic coincidence and the high voltage fixed firing time; a typical value is $\sim 1 \mu \mathrm{s}$.

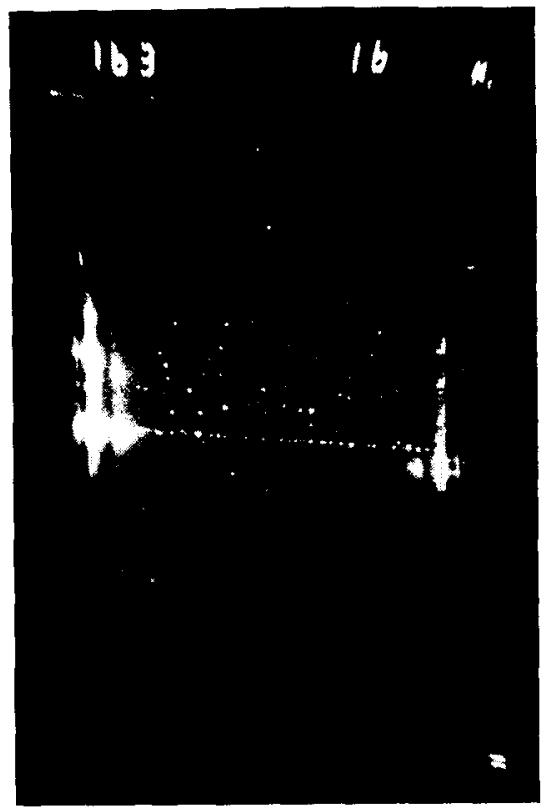

Fig. 24. Track in pure hydrogen at 405 torr; $25 \Omega$ chamber, window $35 \times 55 \mathrm{~cm}^{2}$.

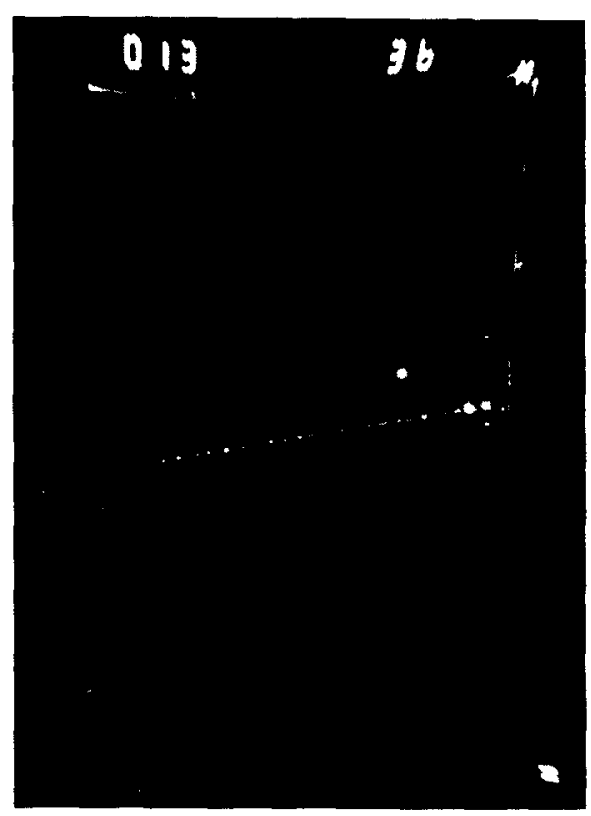

Fig. 25. Track in the best $\mathrm{H}_{2}$ mixture found: $\mathrm{H}_{2}+0.5 \% \mathrm{CH}_{4}+$ $+0.25 \mathrm{ppm} \mathrm{SF}_{6}$ at 385 torr; $25 \Omega$ chamber, window $35 \times 55 \mathrm{~cm}^{2}$. 
A simple way to adjust the memory time is to mix in $\mathrm{H}_{2}$ an electronegative impurity which can attach the primary electrons produced along the track. For this purpose $\mathrm{SF}_{6}$ is used in very small quantities. The memory of the mixture is measured by counting the streamer density $\delta_{\mathrm{s}}$ as function of the delay $\tau_{\mathrm{d}}$ between the passage of the cosmic ray and the application of the high voltage to the streamer chamber $\left.\left[\delta_{s}=\delta_{0} \exp -\lambda_{\tau_{\mathrm{d}}}\right)\right]$. By plotting $\log \delta_{\mathrm{s}}$ as a function of $\tau_{\mathrm{d}}$ straight lines are observed; the measure of their negative slope gives the value of the gas mixture memory. Table 7 and fig. 26 show the main results obtained.

These results can be expressed into the following linear relationship: $\lambda^{-1}=k x+\lambda^{-1}$ with

$\lambda_{0}^{-1}=(0.018 \pm 0.003) \mu \mathrm{s}^{-1}$,

$k=(1.16 \pm 0.10) \mu \mathrm{s}^{-1} \cdot \mathrm{ppm}$.

For $1 \mu \mathrm{s}$ memory $\sim(0.85 \pm 0.1) \mathrm{ppm}$ of $\mathrm{SF}_{6}$ are enough. The streamer density with $0.95 \mu$ s is also given. The streamer density at $1 \mu \mathrm{s}$ does not lie on the straight portion of the $\log \delta_{\mathrm{s}}=f\left(\tau_{\mathrm{d}}\right)$ plot for the long memory mixtures but is always below the expected value. This is likely due to low energy delta rays in the gas (a few tens of $\mathrm{keV}$ ) which gives rise from time to time to clusters which are counted for only one streamer. The extrapolated value of the streamer density for zero time delay gives $\delta_{0}=(2.0 \pm 0.1) \mathrm{cm}^{-1}$ at 380 torr and $\delta_{0}=(3.5 \pm 0.3) \mathrm{cm}^{-1}$ at 725 torr. (From ref. 14 the primary ionisation of $\mathrm{H}_{2}$ at $20^{\circ} \mathrm{C}$ and 725 torr should be $4.53 \mathrm{~cm}^{-1}$.)

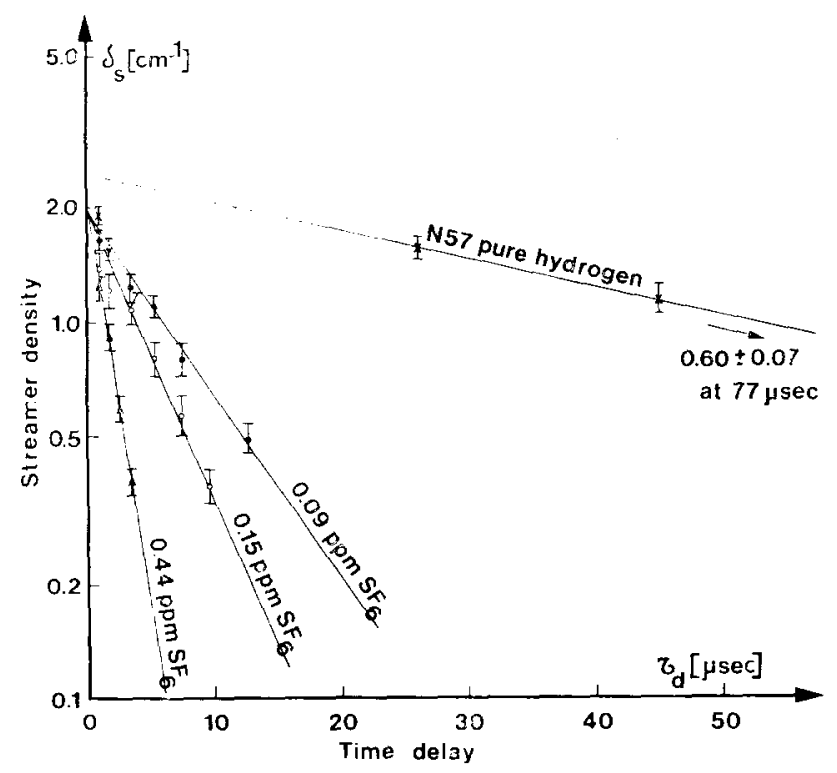

Fig. 26. Hydrogen memory at 380 torr.
TABle 7

Hydrogen memory with adjunction of $\mathrm{SF}_{6}$ at 380 torr.

\begin{tabular}{cccc} 
Gas & $\begin{array}{c}\times \mathrm{SF}_{6} \\
(\mathrm{ppm})\end{array}$ & $\begin{array}{c}\lambda^{-1} \text { memory } \\
(\mu \mathrm{s})\end{array}$ & $\begin{array}{c}\delta_{s}(0.95 \mu \mathrm{s}) \\
\text { at } 380 \text { torr }\end{array}$ \\
\hline $\begin{array}{c}\text { pure } \mathrm{H}_{2} \\
\mathrm{H}_{2}+0.5 \% \mathrm{CH}_{4}\end{array}$ & $0.09 \pm 0.03$ & $9.6 \pm 1.2$ & $1.67 \pm 0.12$ \\
$\mathrm{H}_{2}+0.5 \% \mathrm{CH}_{4}$ & $0.15 \pm 0.03$ & $5.5 \pm 0.7$ & $1.35 \pm 0.18$ \\
$\mathrm{H}_{2}+0.5 \% \mathrm{CH}_{4}$ & $0.44 \pm 0.03$ & $1.9 \pm 0.3$ & $1.21 \pm 0.08$ \\
\hline
\end{tabular}

\subsection{VARIOUS RESULTS AND EFFECTS}

Using a $\pm 18^{\circ}$ stereo angle, the RCA and the EMI devices have been compared with a $\times 20$ demagnification. EMI with $F 5.6$ gives about the same track brightness as RCA with F 3.5. With a same $F$ number (3.5) the voltage for obtaining good tracks in the chamber with the EMI is $\sim 2 \%$ smaller. These results agree with the $\times 3$ difference in light gain between the two image intensifiers.

For a given value of the peak field in the chamber filled with $\mathrm{He}-\mathrm{Ne}$ it has been found that the pressure can be increased by $\sim 20 \%$ between direct photography and pictures obtained using the RCA image intensifier. This corresponds to $\sim 13 \%$ decrease in the peak field for a given pressure. A $13 \%$ increase in field implies a $\sim 10^{4}$ increase in brightness which is close to the gain which is expected from the RCA intensifier.

The effect of the length of the pulse on the track

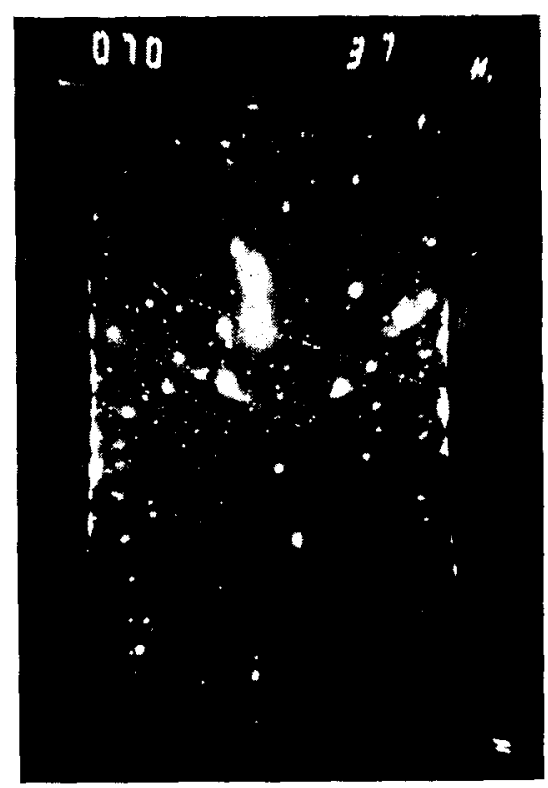

Fig. 27. $\mathrm{H}_{2}+\frac{1}{2} \% \mathrm{CH}_{4}$ before coating of the central high voltage cathode mesh (660 torr); $25 \Omega$ chamber. 
quality has been investigated. Keeping all the streamer chamber conditions the same, a $\tau_{1 / 2}=6.0 \mathrm{~ns}$ has been used instead of the standard $4.0 \mathrm{~ns}$ (see table 2). This test has been made in the $9 \mathrm{~cm}$ gap chamber filled with $\mathrm{H}_{2}\left(+\frac{1}{2} \% \mathrm{CH}_{4}\right)$ at 520 torr by making a comparison between the average length of the streamers obtained with the two different pulses. The result is that for a given brightness the average length of the streamers increases when the duration of the pulse increases With the 6 ns pulse the peak amplitude necessary for getting good tracks is decreased by $\sim 14 \%$ compared to the $4 \mathrm{~ns}$ pulse. This is in good agreement with the $\hat{E}^{3} \tau_{1 / 2}=$ const. law. It has also been found that glows or parasitic sparking was much more often observed with the long pulse ( 35 out of 38 pictures with tracks with the long pulse compared to 17 out of 56 in the case of the short one). This confirms the original thought that the shorter the pulse the better is the track quality.

The very important improvement of the insulation property of the central mesh against field emission must again be emphasized. This was a major step for getting good tracks at atmospheric pressure in $\mathbf{H}_{2}$. A comparison between fig. 27 and figs. 28 and 29 is enough by itself to prove how important was this technical improvement.

\section{Conclusions}

In $1971^{1}$ ) it was possible to conclude from the

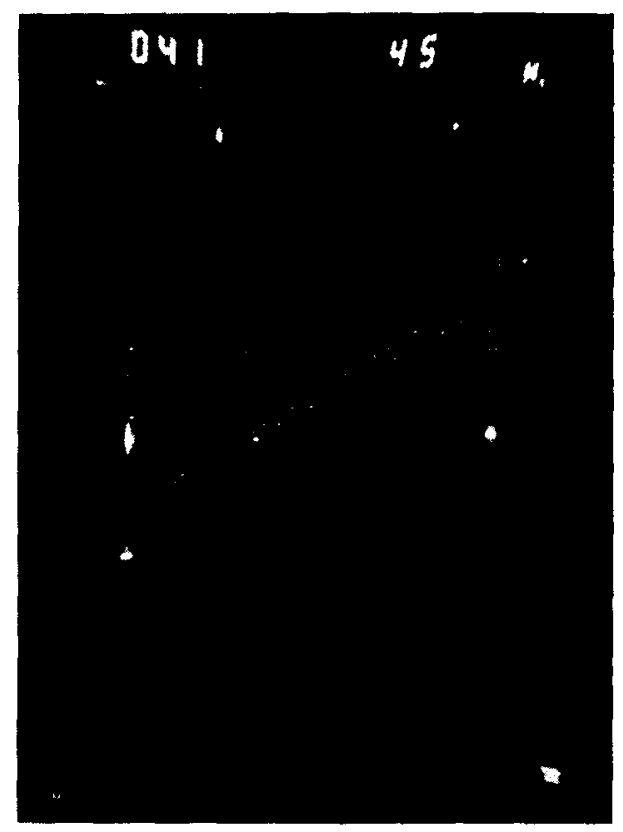

Fig. 28. $\mathrm{H}_{2}+\frac{1}{2} \% \mathrm{CH}_{4}$ after coating of the central high voltage cathode mesh; $25 \Omega$ chamber, 735 torr. previous test ${ }^{2}$ ) with direct photography that a full scale $2 \times 10$ to $2 \times 15 \mathrm{~cm}$ gap $\mathrm{H}_{2}$ chamber could be built and made to work satisfactorily. We can now say that thanks to the use of an image intensifier and to the discovery of various technical improvements a $2 \times$ $\times 10 \mathrm{~cm}$ gap $\mathrm{H}_{2}$ chamber has worked satisfactorily giving thousands of pictures up to atmospheric pressure in $\mathrm{H}_{2}$ with a good quality for making precise reconstruction of events. This was a major advance towards the use of this instrument in high energy physics.

There are still some technical difficulties to be solved mainly to prevent sparking at the entrance of the beam channel in the chamber and to increase the picture efficiency by reducing the pulse amplitude jitter. An experiment with a million pictures could then be envisaged bearing in mind that the long term reliability has not yet been experienced.

A combination of a conventional $\mathrm{He}-\mathrm{Ne}$ chamber (with a liquid $\mathrm{H}_{2}$ target) with a new $\mathrm{H}_{2}$ streamer chamber gas target would have so many attractive properties that it appears as a very powerful, flexible and reasonably cheap vertex detector for the high energy physics of the future.

We wish to thank A. Lagarrigue, A. Minten, D. Morellet, P. Musset, C. Peyrou and A. Rousset for their interest and support in this work.

We are particularly grateful to the technical assis-

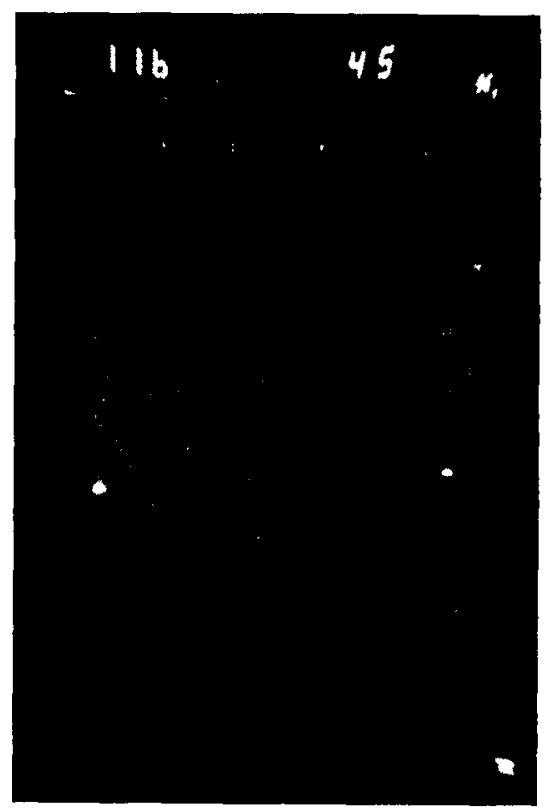

Fig. 29. $\mathrm{H}_{2}+\frac{1}{2} \% \mathrm{CH}_{4}$ after coating of the central high voltage cathode mesh; $25 \Omega$ chamber. 735 torr. 
tance of Messrs. H. Cabel, G. Guilhem and J. Leroy for their essential work. We also thank S. Hajdas, $H$. Vercez from Orsay and all the excellent technical services from TC-L group at CERN.

We would like to thank K. Eggert, E. Gygi and F. Schneider for making available to us the image intensifiers and for their assistance in the operation.

We also wish to thank P. Heusse and Prof. C. GreyMorgan for many interesting discussions concerning this work.

\section{References}

1) F. Rohrbach, J. J. Bonnet, M. Cathenoz, Nucl. Instr. and Meth. 111 (1973) 485.

2) B. C. Tan, H. Schmied, A. Rousset, F. Rohrbach, F. Piuz, F. Gross, C. Grey-Morgan et M. Cathenoz, CERN TC-L/Int. 70-7 (CERN, 1970).

3) V. Cook, R. Gregg, A. M. Jonckheere. R. Kenyon, H. J.
Lubatti and F. Rohrbach, Proc. Ist Int. Conf. on Streamer chamber technology, ANL-8055, (Argonne National Laboratory, 1972).

4) LPNHE, Ecole Polytechnique, Paris, LAL Orsay, Int. Conf. on Nuclear physics instrumentation, Frascati (1973).

5) F. Rohrbach, Int. Conf. on Nuclear physics instrumentation Frascati (1973).

6) F. Rohrbach, CERN 71-28 (Yellow Report) (1971) p. 56.

$\left.{ }^{7}\right)$ P. Felsenthal and J. M. Proud, Phys. Rev. 139 (1965) A1796.

$\left.{ }^{8}\right)$ I. V. Falomkin, M. M. Kulynkin, G. B. Pontecorvo and $\mathrm{Yu}$. A. Sherbakov, Colloq. Int. l'Electronique Nucléaire, vol. 3 (Versailles, 10-13 Sept. 1968) p. 8-1.

$\left.{ }^{9}\right)$ H. Gentsch, E. Gygi, M. Hanney and F. Schneider, CERN 74-4 (Yellow Report) (1974).

10) K. Eggert et al., Nucl. Instr. and Meth. 126 (1975) 477.

11) A. J. Davies, C. J. Evans and C. Grey-Morgan, CERN TC-L/Int. 71-1 (1971).

12) A. J. Davies, C. J. Evans, CERN 73-10 (Yellow Report) (1973).

13) V. Eckardt, Thesis (Hamburg Universität, 1971).

14) V. K. Ermilova, L. P. Kotenko, G. I. Merzon and V. A. Chechin, Sov. Phys. JETP 29, no. 5 (1969). 